\title{
Jeotermal Rezervuarlarla İlgili Sayısal Modelleme/Benzeşim Çalışmaları
}

\author{
Numerical Modeling/Simulation Studies of the Geothermal Reservoirs \\ Nurkan KARAHANOĞLU D \\ Üniversiteler Mahallesi, Dumlupınar Bulvarl, No 1, Orta Doğu Teknik Üniversitesi \\ Jeoloji Mühendisliği Bölümü, Ankara
}

Geliş (Received): 10 Aralık (December) 2018 / Düzeltme (Revised): 13 Ocak (January) 2019 / Kabul (Accepted): 14 Ocak (January) 2019

\section{ÖZ}

Jeotermal rezervuarlar içerdikleri yüksek basınç ve yüksek entalpi nedeniyle çok kıymetli yenilenebilir enerji kaynağı olarak değerlendirilmektedir. Doğal süreçler sonucu rezervuar kayaçta depolanan jeotermal akışkan elektrik enerjisi üretiminde, konut 1sıtmacılığında, seracılıkta ve balneolojik amaçlı olarak kullanılmaktadır. Jeotermal rezervuarların üretimi doğal dengede bulunan hidrodinamik ve hidrotermal mekanizmaları harekete geçirmekte ve bunun sonucu olarak rezervuar içinde akışkan hareketi ve 1sı yayılımı/taşınımı oluşmaktadır. Bu mekanizmaların matematiksel olarak tanımlanması ile başlayan sayısal modelleme çalışmaları değişik işletim koşullarının rezervuara etkisini araştırabilmekte ve dolayısı ile jeotermal sistemlerin sürdürülebilir düzeyde üretilmelerine olanak sağlamaktadır. Söz konusu mekanizmaların diferansiyel denklemlerle ifade edilmesi ve jeotermal rezervuarın kavramsal modeline dayalı olarak belirlenen fiziksel parametreler ve uygun sınır koşullarının uygulanması sayısal modelleme çalışmalarının temelini oluşturmaktadır. 1970 li yıllardan beri sürdürülen modelleme çalışmaları ile dünya genelinde birçok jeotermal sistemde sayısal modelleme çalışması yapılmış ve bu rezervuarların en uygun ve sürdürülebilir üretim ve işletim politikalarının geliştirilmesi sağlanmıştır. Bu makalede jeotermal rezervuarların sayısal modellenmesinin temel prensipleri anlatılmakta, ilgili denklemler tanıtılmakta ve tarihsel gelişim aktarılmakta ve hazır paket programlar olarak sunulan simulatörlerin/modellerin gizemleri açıklanmaktadır. Ayrıca sayısal model çalışması yapılan sahalar ve ilgili ülkeler tanıtılarak bu çalışmaların kaynakları verilmektedir.

Anahtar Kelimeler: Jeotermal rezervuarlar, Sayısal modelleme/Benzeşim, Simülatörler

\begin{abstract}
Geothermal reservoirs are renewable energy resources and they are treated as very valuable due to their high pressure and high enthalpy contents. Geothermal fluid stored in the reservoir is used for electricity production, central heating, greenhouse heating and for balyneological purposes. Fluid production from such reservoirs triggers hydrodynamic and hydrothermal mechanisms and causes fluid movement and heat transfer in the reservoirs. Modeling studies start with defining these mechanisms by differential equations and help investigating response of reservoirs to alternative production scenarios and hence obtain sustainable management of such systems. Fundamental studies for geothermal reservoir simulation require formulation of the mechanisms and application of boundary and initial conditions and the physical parameters obtained from conceptual model of the geothermal system. Geothermal reservoir modeling studies date back to 1970s and several geothermal reservoirs in the world have been simulated to determine optimum and sustainable production policies. This paper summarizes the basic principles of geothermal
\end{abstract}


Karahanoğlu

reservoir modeling and introduces basic differential equations and explains mysteries of the reservoir simulators nowadays widely used in the geothermal industry. The numerically simulated geothermal fields and the related countries are also summarized together with the references.

Keywords: Geothermal reservoirs, Numerical modeling/Simulation, Simulators

\section{GİRIŞ}

Jeotermal sistemler doğal süreçler sonucu oluşan ve insanlık tarafından öncelikle kaplıca amaçlı ve daha sonraki yıllarda içerdigi yüksek basınç ve entalpi zenginliğinin farkedilmesiyle enerji üretimi amaçlı kullanılan yenilenebilir enerji kaynaklarıdır. Bu sistemlerin hidromekanik ve hidrotermal özelliklerinin tanımlanması, rezervuar kapasitelerinin belirlenmesi ve en uygun koşullarla üretilerek sürdürülebilir olarak değerlendirilmeleri açısından son derece önem arz etmektedir.

Jeotermal rezervuarların üretime açılması jeolojik zaman içinde dengeye ulaşan hidrodinamik ve hidrotermal mekanizmaların harekete geçmesine ve üretim koşullarına bağlı olarak basınç ve sicaklık düşümlerine neden olmaktadır. $\mathrm{Bu}$ durum gerçek kapasitesi tam olarak belirlenmemiş ve reşarj/deşarj dengesi kurulamamış rezervuarlarda jeotermal sistemin kaybedilmesine yol açabilmektedir. Jeotermal rezervuarların üretilmeleri esnasında sicaklık ve basınç düşümleri ile belirlenen olumsuz gelişmeler bilimsel çalışmalar yapılmasını ve sistemin dengede tutulabilmesi için en uygun üretim koşullarının belirlenmesini gerekli kılmıştır. $\mathrm{Bu}$ amaçla yapılan araştırmalar jeotermal sistemde gelişen mekanizmaların matematiksel olarak tanımlanmasını sağlamış ve bilgisayar teknolojisindeki gelişmelerle birlikte sayısal modelleme konusunda çalışmalar yapılmasının önü açılmıştır.

1970 li yıllarda başlayan sayısal modelleme çalışmaları ile jeotermal sahaların gerçek kapasitelerinin belirlenmesi ve en uygun üretim ve enjeksiyon koşulları ile değerlendirilmeleri ve sürdürülebilir olarak üretilmeleri sağlanmıştır. Jeotermal sahaların sayısal modellemesi konusunda çok sayıda araştırma yapılmış ve sayısal modellemede kullanılan programların geliştirilmeleri yanında sayısal modellerin jeotermal sahalara uygulanması yönünde çok değerli bilimsel birikimler elde edilmiştir.

$\mathrm{Bu}$ makalede jeotermal sistemlerin sayisal modellemesi konusunun önemi vurgulanarak bu konuda yapılan bilimsel araştırmaların ayrıntıları, tarihsel gelişimi ve dünya genelinde yapılan modelleme çalışmaları ve sayısal modellemede kullanılan programlar sunulmaktadır. Diğer taraftan jeotermal rezervuarların sayısal modellenmesi konusunun temel prensiplerinin tanıtıldığ 1 bu araştırmada ülkemizde yapılan modelleme çalışmaları hakkında bilgiler verilmektedir.

\section{TARİHSEL GELIŞ̧iM}

1970 li yıllarda bilgisayar teknolojisindeki hızlı gelişmelerle birlikte araştırmacılar jeotermal rezervuarların sayısal modellemesi konusuna yönelmişler ve araştırmalarını jeotermal rezervuarların optimum ve sürdürülebilir işletimini sağlamak için geliştirdikleri sayısal modeller üzerinde yoğunlaştırmışlardır. $\mathrm{Bu}$ konuda Gupta vd. (1974), Mercer vd. (1974), Faust ve Mercer (1975), Garg vd. (1975), Lasseter (1976), Bodvarsson vd. (1982), Pruess vd. (1982) yaptıkları araştırmalar öncü çalışmalar olarak değerlendirilmektedir. $\mathrm{Bu}$ çalışmalarda 
kütlenin ve enerjinin korunumu prensiplerinin Darcy kanunu ile birleştirilerek jeotermal sistemlerdeki mekanizmaların matematiksel olarak ifade edildikleri görülmektedir. $\mathrm{Bu}$ y1llarda geliştirilen matematiksel modellerde kütle denklemleri ile enerji denklemleri birbirleri ile tam iletişimli (fully coupled) olarak oluşturulmuş ve gerek gözenekli ortamdaki akışkan (tek fazlı/iki fazlı) ve gerekse fiziksel parametreler için (değişkenlerden bağımsız/ değişkenlere bağımlı) değişik varsayımlar kabul edilmiştir. Rezervuar ortamında gelişen akışkan hareketi ve enerji yayılımı mekanizmaları için model denklemlerinin basınç ve entalpi/ sıcaklık değişkenleri için oluşturulması ve Sonlu Elemenlar/Sonlu Farklar kullanılarak sayısal olarak çözülmeleri birçok araştırmacı tarafindan başarılı olarak uygulanmıştır (Brownell vd., 1977; Toronyi ve Farouq Ali, 1977; Ertekin, 1978; Thomas ve Pierson, 1978; Faust ve Mercer, 1979a; Faust ve Mercer, 1979b; Morris ve Campbell, 1981; Bodvarsson vd., 1981; Carradori vd., 1981; Nguyen ve Pinder, 1983; Zyvoloski, 1983; Karahanoğlu vd., 1984; O'sullivan, 1985; Bodvarsson vd., 1986). Örnek olarak Mercer ve Faust tarafından geliştirilen matematiksel modelin Yeni Zellanda'daki Wairakei jeotermal sistemine uygulanışı ve değişkenlerin (basınç ve entalpi) gözlenen değerleri ile modelden elde edilen sonuçların birbirleri ile uyumlu olduklarından bahsedilebilir (Mercer ve Faust, 1979). Bu dönemde geliştirilen sayısal modellerin değişik ülkelerdeki jeotermal sahalara uygulandığ 1 ve jeotermal sistemlerin sayısal modeller yardımı ile değerlendirildikleri ve geliştirildikleri izlenmektedir (Bodvarsson vd., 1987a; Bodvarsson vd., 1987b; Aunzo vd., 1989; Yasukawa ve Ishido, 1990; Pruess, 1990; O’Sullivan vd., 1990; Menzies vd., 1991; Axelsson ve Bjornsson, 1993; Sakagawa vd., 1994; Mc.Guinness vd., 1995; Arnorsson,
1995; Parini vd., 1995a, b; Boardman vd., 1996; Hanano vd., 1998). Takip eden y1llarda üretime bağlı olarak gelişen yüzey çökmesi problemi konusunda da çalışmalar yapılmış ve hazırlanan sayısal modeller başarılı olarak uygulanmıştır (Lipmann vd., 1976; Morgan vd., 1980; Lewis ve Karahanoğlu, 1981; Bear ve Çorapçıoğlu, 1981; Bodvarsson vd., 1981; Aboustit vd., 1985; Karahanoğlu vd., 1984; Lewis vd., 1989; Rutqvist vd., 2002; Hu vd., 2013; Bromley vd., 2013; Jing vd., 2014; Karrech vd., 2015).

1990 l1 yıllardan itibaren jeotermal rezervuarların sayısal modellenmesi konusunda yapılan araştırmalarda, modellerin geliştirilmesi ve jeotermal sahalara uygulanması konusunda hizlı bir artış gözlenmektedir. TOUGH2 programının modelleme çalışmalarında kullanılması bu yıllarda ortaya çıkmış (Çizelge 1) ve Pruess (1990) sunduğu makalesinde jeotermal rezervuarların modellenmesi konusundaki temel prensipleri, modelleme çalışmalarının ayrıntılarını ve saha uygulamaları hakkındaki çalışmaları özetlemekte ve zaman içerisinde yapılması düşünülen araştırmaları anlatmaktadır.

İlk yıllarda tek fazlı (sıcak su tipli) ve sabit fiziksel parametrelerin kullanıldığ 1 sayısal modellerin y1llar içerisinde geliştirildiği, iki fazlı (sıcak su ve buhar) ve faz değişimlerinin dikkate alındığ 1 ve birçok parametrenin basınç ve sıcaklık/entalpi değişkenleri ile ifade edildiği üç boyutlu modellerin jeotermal endüstrisine sunulduğu görülmektedir (Pruess, 1990; Bodvarsson vd., 1990a; Finsterle vd., 1993; McGuinnes vd., 1995; Hadgu vd., 1995; Suarez vd., 1996; Kiryukhin, 1996; Pruess, 1997; Hanano, 1998; Pruess, 1999; Pruess vd., 1999; Mannington vd., 2000; Barmin ve Kondrashov, 2000; Rutqvist vd., 2002; Bataille vd., 2006; Seol ve Lee, 2007; Driesner ve Geiger, 2007; Croucher ve O'Sullivan, 2008; O'Sullivan vd., 
Karahanoğlu

2009; Kumamoto vd., 2009; Blocher vd., 2010; Emoricha vd., 2010; Cerminara ve Fasano, 2012; Jiang vd., 2013; Guerrero-Martinez ve Verma, 2013; Moridis ve Freeman, 2015).

Zaman içerisinde yapılan araştırmalarda sayisal modellerin jeotermal sahaların geliştirilmeleri ve optimum ve sürdürülebilir olarak değerlendirilmesi için kullanıldıkları izlenmektedir (Atmojo vd., 2000; Sakagawa vd., 2000; Portugal vd., 2000; Fendekova ve Fendek, 2000; Battistelli vd., 2002; Arellano vd., 2003; Mannington vd., 2004; Fabbri ve Trevisani, 2005; Zarrouk vd., 2007; Porras vd., 2007; Vedova vd., 2008;; Arias vd., 2010; Yahara ve Tokita, 2010; Kiryukhin vd., 2010; Itoi vd., 2010; Barelli vd., 2010a, Barelli vd., 2010b; Noorallahi ve Itoi, 2011; Gunnarsson vd., 2012; Zaher vd., 2012; Lei ve Zhu, 2013; Pearson vd., 2014; Canet vd., 2015; Farkhutdinov vd., 2015; Bujakowski vd., 2016; Zhang vd., 2016; Turali ve Şimşek, 2017). Bu çalışmalar arasında Stanford Üniversitesi Lawrence Berkeley Laboratuvar1 ekibinin yaptığı araştırmalar ve program yönündeki çalışmalar önemli bir yer tutmaktadır. $\mathrm{Bu}$ çalışmaların ürünü olarak Finsterle (1993) iTOUGH2 programının kullanım klavuzunu 1993 yılında sunmuş (Finsterle, 1993) ve programın ikinci versiyonu 1999 yilında Pruess tarafından tanıtılmıştır (Pruess vd., 1999). Takip eden yıllarda bu programın geliştirilmesi yönündeki araştırmaları ve jeotermal sahaların modellenmesi yönündeki çalışmaları O'Sullivan vd. (2001) hazırladıkları makalede sunmuşlar ve dünya genelinde yapılan modelleme çalışmalarını ülkeler bazında Çizelge halinde vermişlerdir. Benzer şekilde Franco ve Vaccaro (2014) örnek modelleme olarak Momotombo (Nikaragua), Ngatamariki ve Wairakei (Yeni Zellanda), Larderello ve Mt. Amiata (İtalya), Groß Schönebeck (Almanya) jeotermal sahalarında yapılan çalışmaları ayrıntılı olarak anlatmışlar ve birçok jeotermal sahada yapılan sayısal modelleme çalışmalarının ayrıntılarını (modellenen dönem, uygulanan program, sahanın geometrisi, hazırlanan grid sistemi, koşullar ve fiziksel parametreler ve kalibrasyon çalışmaları) liste halinde sunmuşlardır.

Ayrıntılı ve çok değerli bilgiler içeren bu listelerde göze çarpan en önemli noktanın, ilk y1llarda ticari olmayan programların/ simülatörlerin kullanıldığı ve tarihsel süreç içerisinde serbest kullanıma kapalı TOUGH programının etkin bir şekilde tercih edilmiş olduğu görülmektedir. Son yıllarda yapılan çalışmalar ve araştırmalar eklenerek bu listeler güncelleştirilmiş ve yeni hali ile Çizelge 1 de sunulmuştur.

2003 yılında TOUGH yazılımı konulu bir sempozyum düzenlenmiş ve TOUGH programının değişik modülleri tanıtılarak diğer programlarla karşılaştırılması yapılmıştır (Pruess, 2003).

Üç yılda bir tekrarlanan sempozyumlarda TOUGH programı ile yapılan çalışmalar sunulmakta ve program ile ilgili yeni gelişmeler, değişik araştırmalar paylaşılmaktadır. $\mathrm{Bu}$ tür sempozyumlarda TOUGH programinın uygulandığı karmaşık jeolojik ortamlarda yeraltısuyunun ve ısının hareketini araştıran çalışmalar sunulmaktadır. $\mathrm{Bu}$ araştırmalar yeraltısuyu hareketi, jeotermal rezervuar mühendisliği, çevresel değerlendirme çalışmaları, nükleer atık depolanması, $\mathrm{CO}_{2}$ depolanmas1, petrol ve gaz üretimi, ve hidrojeolojik, biyojeokimyasal, termal, ve jeomekanik süreçlerin bulunduğu tam etkileşimli problemleri kapsamaktadır.

Finsterle vd. (2012) ve Finsterle vd. (2014) TOUGH programı ile ilgili olarak, jeotermal endüstrisinden ve kamu kurumlarından gelen istekler doğrultusunda yapılan gelişmeleri 
anlatmakta ve programın çok karışık problemleri çözebilecek şekilde geliştirildiğini ve karmaşı yeraltı proseslerini modelleyecek özelliklere kavuşturulduğunu iletmektedir. Birinci makalede TOUGH programının gelişimi hakkında bilgiler verilmekte ve $\mathrm{TOUGH}+$, TOUGHREACT, TOUGH2, iTOUGH2 kodlarının teknik özellikleri anlatılmaktadır. Her iki makalede yaygın olarak kullanılan TOUGH simulatörlerinin isimleri, ve proseslerini tanıtan bir Çizelge sunulmaktadır (Çizelge 2). Temel denklem sistemlerinin ve bu denklemlerin sonlu farklar yöntemi ile çözülmelerinin korunduğu ana yazılımda yıllar içerisinde iyileştirmeler yapıldığı, yeni durum denklemlerinin geliştirildiği ve programın çoklu fiziksel kapasitesinin genişletildiği ve sayısal performansını artırıcı gelişmelerin elde edildiği ve kuyu iletişimi, yüzey sularıyla ilişkiler ve atmosfer ile olan ilişkiler sağlayan modüllerin programa bağlandığı anlatılmaktadır. Çizelge 2 ilgili sempozyumda sunulan çalışmaların TOUGH programının geliştirilmesine katk1 sunduğu konuları ayrıntılı olarak vermektedir. Ayrıca son gelişmeler konusu ile ilgili olarak, 'durum denklemi', 'reaktif taşınım', 'kaya mekaniğii', 'ters modelleme' ve diğer konularda yapılan gelişmeler anlatılmaktadır.

Çizelge 1. Jeotermal sahaların sayısal modellenmesi konusundaki çalışmalar (O’Sullivan vd., 2001 ve Franco ve Vaccaro, 2014'den düzenlenmiş ve güncelleştirilmiştir).

Table 1. Numerical modeling studies of Geothermal fields (modified and revised from O'Sullivan et al., 2001 and Franco and Vaccaro, 2014).

\begin{tabular}{|c|c|c|c|}
\hline Simulator & Year & Author or Company & Field Country \\
\hline Program & Y1l & Yazar veya Şirket & Saha Ülke \\
\hline & 1979 & Gupta vd., 1979 & Puga Hindistan \\
\hline & 1979 & Mercer ve Faust, 1979 & Wairakei, Yeni Zellanda \\
\hline SHAFT 78 & 1979 & Pruess vd., 1979 & Model rezervuar \\
\hline \multirow[t]{3}{*}{ SHAFT79 } & 1982 & Bodvarsson vd., 1982 & Olkaria Kenya \\
\hline & 1984 & Karahanoğlu vd., 1984 & Kızıldere Türkiye \\
\hline & 1985 & Ingebritsen ve Sorey, 1985 & Lassen, Hollanda \\
\hline \multirow[t]{5}{*}{ HYDRO THERM } & 1985 & Ingebritsen ve Sorey, 1985 & Lassen, ABD \\
\hline & 1987 & Bodvarsson vd., 1987a & Olkaria, Kenya \\
\hline & 1988 & Bodvarsson, 1988 & Svartgensi, İzlanda \\
\hline & 1989 & Aunzo vd., 1989 & Ahuachapan, El Salvador \\
\hline & 1990 & Yasukawa ve Ishido, 1990 & Onikobe, Japonya \\
\hline TOUGH2 & 1990 & O’Sullivan vd., 1990 & Kamojang, Endonezya \\
\hline TOUGH2 & 1990 & Antunez vd., 1990 & Mofete, İtalya \\
\hline TOUGH2 & 1990 & O’Sullivan vd., 1990 & Kamojang, Endonezya \\
\hline THOR & 1990 & Yasukawa ve Ishido, 1990 & Onikobe Caldera, Japonya \\
\hline TOUGH2 & 1990 & Bodvarsson vd., 1990a & Nesjavellir, İzlanda \\
\hline TS\&E & 1990 & Williamson, 1990 & Geysers, ABD \\
\hline TOUGH2 & 1990 & Sanyal vd., 1990 & Onikobe, Japonya \\
\hline
\end{tabular}


Karahanoğlu

\begin{tabular}{|c|c|c|}
\hline TOUGH2 & 1990 & Bodvarsson vd., 1990b \\
\hline TOUGH2 & 1991 & Antunez vd., 1991 \\
\hline STAR & 1991 & Pritchett vd., 1991 \\
\hline TOUGH2 & 1991 & Menzies vd., 1991 \\
\hline TOUGH2 & 1991 & Ripperda vd., 1991 \\
\hline TOUGH2 & 1991 & Aunzo vd., 1989 \\
\hline TOUGH2 & 1992 & Battistelli vd., 1992 \\
\hline TOUGH2 & 1992 & Burnell, 1992 \\
\hline TOUGH2 & 1993 & Amistoso vd., 1993 \\
\hline TOUGH & 1992 & Hanano, 1992 \\
\hline TETRAD & 1993 & Strobel, 1993 Bulalo, \\
\hline TOUGH2? & 1993 & Axelsson ve Bjornsson, 1993 \\
\hline TOUGH2? & 1995 & Pham vd., 1995 \\
\hline TOUGH2 & 1994 & Antunez vd., 1994 \\
\hline SING II & 1994 & Sakagawa vd., 1994 \\
\hline SING I, SING II & 1995 & Nakanishi vd., 1995 \\
\hline TOUGH2 & 1995 & Todesco, 1995 \\
\hline TOUGH2 & 1995 & McGuinness vd., 1995 \\
\hline STARS & 1995 & Arihara vd., 1995 \\
\hline STAR & 1995 & Bertani ve Cappetti, 1995 \\
\hline STAR & 1995 & Yano ve Ishido, 1995 \\
\hline STAR & 1995 & Pritchett ve Garg, 1995 \\
\hline STAR & & Ariki (Mitsubishi Metals) * \\
\hline$?$ & 1995 & Nakanishi vd., 1995 \\
\hline$?$ & 1995 & Tokita vd., 1995 \\
\hline TETRAD & 1995 & Menzies ve Pham, 1995 \\
\hline AQUA & 1995 & $\mathrm{Hu}, 1995$ \\
\hline TOUGH2 & 1996 & Kiryukhin, 1996 \\
\hline TOUGH2 & 1996 & Suarez vd., 1996 \\
\hline GEOSIM6 & 1996 & Pham vd., 1996 \\
\hline TOUGH2 & 1996 & Kissling vd., 1996 \\
\hline \multirow[t]{3}{*}{ TOUGH2 } & 1996 & Parini vd., 1996 \\
\hline & 1996 & Absar vd., 1996 \\
\hline & 1997 & White vd., 1997 \\
\hline TOUGH2 & 1997 & Battistelli vd., 1997 \\
\hline TOUGH2 & 1997 & Antics, 1997 \\
\hline TETRAD & 1997 & Sanchez, 1997 \\
\hline TOUGH2 & 1998 & O’Sullivan vd., 1998 \\
\hline TETRAD & 1998 & Bloomfield vd., 1998 \\
\hline STAR & 1998 & Ishido ve Tosha, 1998 \\
\hline ROCK-FLOW-2 & 1998 & Kolditz ve Klauser, 1998 \\
\hline
\end{tabular}

Olkaria, Kenya

Cerro Prieto, Meksika

Sumikawa, Japonya

Zunil, Guatemala

Ahuachapán, El Salvador

Ahuachapán, El Salvador

Nagqu Tibet, Çin

Rotorua, Yeni Zellanda

Palinpinon, Filipinler

Matsukawa, Japonya

Filipinler

Botn, İzlana

Uenotai, Japonya

Geysers, B

Mori, Japonya

Oguni, Japonya

Vulcano, İtalya

Kakkonda, Japonya

Kakkonda, Japonya

Monteverdi, Italya

Kirishima, Japonya

Oguni, Japonya

Oguni, Japonya

Copahue, Arjantin

Hatchobaru, Japonya

Geysers, ABD

Yangbajian Tibet, Çin

Dachny, Rusya

Los Azufres, Meksika

Amatitlán, Guatemala

Wairakei, Yeni Zellanda

Miravelles, Kosta Rika

Puga Hindistan

Kawerau, Yeni Zellanda

Skierniewice, Polonya

Oradea, Romanya

Los Humeros, Meksika

Wairakei, Yeni Zellanda

Cove Fort Sulphurdale, ABD

Ogiri, Japonya

Rosemanowes, İngiltere 


\begin{tabular}{|c|c|c|}
\hline TOUGH2 & 1998 & Battistelli vd., 1998 \\
\hline TOUGH2 & 1998 & Antics, 1998 \\
\hline TOUGH2 & 1999 & Sigurdsson * \\
\hline TETRAD & 1999 & Esberto ve Sarmiento, 1999 \\
\hline TETRAD & 1999 & Sta.Ana vd., 1999 \\
\hline ROCK-FLOW-3 & 1999 & Kaiser * \\
\hline TOUGH2 & 1999 & Bjornsson * \\
\hline TOUGH2 & 1999 & Bjornsson, 1999 \\
\hline GEO-CRACK 2D & 1999 & Swenson vd., 1999 \\
\hline STAR & 2000 & Nakanishi ve Iwai, 2000 \\
\hline TOUGH2 & 2000 & Antics, 2000 \\
\hline TOUGH2 & 2000 & Sanyal vd., 2000 \\
\hline AQUA & 2000 & Fendek, 2000 \\
\hline TOUGH2 & 2000 & Atmojo, 2000 \\
\hline QUA & 2000 & Fendekova ve Fendek, 2000 \\
\hline TETRAD & 2000 & Butler vd., 2000 \\
\hline IGS3C & 2000 & Sakagawa vd., 2000 \\
\hline OUGH2 & 2004 & Mannington vd., 2004 \\
\hline TOUGH, ITIUGH2 & 2005 & Porras vd., 200 \\
\hline SHEMAT & 2005 & Köhn ve Stöfen, 2005 \\
\hline ITOUGH2, TOUGH2 & 2006 & Kiryukhin vd., 2006 \\
\hline ITOUGH2 & 2007 & Porras vd., 2007 \\
\hline SUTRA & 2007 & Özkaya, 2007 \\
\hline ITOUGH2 & 2008 & Kiryukhin vd., 2008 \\
\hline SHEMAT 7.1 & 2008 & Vedova vd., 2008 \\
\hline ITOUGH2, MINC Model & 2009 & Kumamotoa vd., 2009 \\
\hline FEFLOW & 2009 & Sonney ve Vuataz, 2009 \\
\hline FEFLOW & 2010 & Blöcher vd., 2010 \\
\hline FEFLOW & 2010 & Magri vd., 2010 \\
\hline MINC Model & 2010 & Itoi vd., 2010Ogiri, \\
\hline TOUGH2 & 2010 & Emoricha vd., 2010 \\
\hline TOUGH2 & 2010 & Romagnoli vd., 2010 \\
\hline TOUGH2 & 2010 & Barelli vd., 2010a \\
\hline TOUGH2 & 2010 & Barelli vd., 2010b \\
\hline TOUGH2 & 2011 & Gunnarsson vd., 2011 \\
\hline HYDROTHERM 2.2 & 2011 & Zaher vd., 2011 \\
\hline SHEMAT & 2011 & Mottagy vd., 2011 \\
\hline DROTHERM 2.2 & 2012 & Zaher vd., 2012 \\
\hline
\end{tabular}

Tendaho, Etyopya

Nagyzszenas, Macaristan

Krafla, İzlanda

Mt. Apo, Filipinler

Tongonan, Filipinler

Soultz-sous-Forets, Fransa

Reykjanes, İzlanda

Svartsengi, İzlanda

Hijiori, Japonya

Onikobe, Japonya

Tomnatic, Romanya

Wasabizawa, Japonya

Galanta, Slovak Cumhuriyeti

Sibayak, Endonezya

Horna Nitra, Slovak

Cumhuriyeti

Cerro Prieto, Meksika

Kakkonda Japonya

Wairakei-Tauhara,

Yeni Zellanda

5Momotombo, Nikaragua

Waiwera, Yeni Zellanda

Pauzhetsky, Rusya

Momotombo, Nikaragua

Kızıldere, Türkiye

Pauzhetsky, Rusya

Larderello, Italya

Ogiri, Japonya

Lavey-les-Bains, İsviçre

Groß Schönebeck, Almanya

Seferihisar, Türkiye

Japonya

Mindanao, Filipinler

Larderello-Travale, Italya

Mt. Amiata, Italya

Larderello-Travale, Italya

Hengill, İzlanda

Gulf of Suez, Misir

The Hague, Hollanda

Hammam Faraun hot spring,

Misir 
Karahanoğlu

FEFLOW,GMS

FEFLOW

TCHEMSYS

AUTOUGH2

TOUGH2

TOUGH2

TOUGH2

FRACTSIM-3D

TOUGH2

METIS

Open GeoSys (OGS)

STIMPLAN

METIS

AUTOUGH2,

TOUGH2

TOUGH2

TOUGH2

TOUGH2

TOUGH2

TOUGH2

TOUGH2

TOUGH2

TOUGH2

TOUGH2

TOUGH2

TOUGH2

TOUGH2

TOUGH2

TOUGH2

TOUGH2

TOUGH2

TOUGH2

TOUGH2

TOUGH2

TOUGH2

TOUGH2

TOUGH2

TOUGH2

TOUGH2

TOUGH2
2013 Sippel vd., 2013

2013 Cherubini vd., 2013

2013 G-Martinez ve Verma, 2013

2013 Lei ve Zhu, 2013

2013 Zeng vd., 2013

2013 Bromley vd., 2013

2014 Pearson vd., 2014

2014 Jing vd., 2014

2015 Llanos vd., 2015

2015 Farkhutdinov vd., 2015

2015 Blocher vd., 2015

2015 Zang vd., 2015

2016 Farkhutdinov vd., 2016

2016 Ratouis vd., 2016

2016 Bujakowski vd., 2016

2016 Carlino vd., 2016

2016 Zhang vd., 2016

2017 Zeng vd., 2017

2017 Feng vd., 2017

2017 Turali ve Şimşek, 2017

2018 Quiono ve Zarrouk, 2018

CEL

CEL

U. of Auckland (O'Sullivan) *

U. of Auckland (O'Sullivan) *

U. of Auckland (O'Sullivan) *

Tokita (WestJEC)

U. of Auckland (O'Sullivan) *

ENEL

ENEL

Tokita (WestJEC)

Sato

U. of Auckland (O’Sullivan) *

White *

Tokita (WestJEC)

Tokita (WestJEC)

Kiryukhin

Kiryukhin

Kiryukhin

Kiryukhin vd 2010
Berlin, Almanya

Groß Schönebeck, Almanya

Las Tres Virgenes, Meksika

Guanyao, Çin

Desert Peak, ABD

Wairakei, Yeni Zellanda

Tauranga, Yeni Zellanda

Hijori, Japonya

Habanero, Avustralya

Khankala, Rusya

Grob Schonebeck, Almanya

EGS KD Çin

Khankala, Rusya

Rotorua, Yeni Zellanda

Podhale, Polonya

Mofete İtalya

Chingshui, Tayvan

Yangbajing, Tibet Çin

Pisa Plain, İtalya

Sorgun, Türkiye

Ngatamariki, Yeni Zellanda

Ahuachapán, El Salvador

Berlin, El Salvador

Darajat, Endonezya

Dieng, Endonezya

Lahendong, Endonezya

Hatchobaru, Japonya

Subiyak, Endonezya

Bagnore, İtalya

Piancastagnaio, İtalya

Otake, Japonya

Yanaizu-Nishiyama, Japonya

Ohaaki, Yeni Zellanda

Tauhara, Yeni Zellanda

Wairakei, Yeni Zellanda

Palinpinon, Filipinler

Malkinsky, Rusya

N-Kurilsky, Rusya

Oceansky, Rusya

Pauzhetsky, Rusya 


\begin{tabular}{|c|c|c|c|}
\hline TOUGH2 & U. of Auckland (O’Sullivan) & $*$ & Mokai, Yeni Zellanda \\
\hline TETRAD & PNOC-EDC & $*$ & Mahanagdong, Filipinler \\
\hline TETRAD & CFE & $*$ & Cerro Prieto, Meksika \\
\hline TETRAD & Unocal & $*$ & Geysers, ABD \\
\hline TETRAD & Bloomfield & $*$ & Geysers, ABD \\
\hline STAR and SING & Ariki (Mitsubishi Metals) & $*$ & Sumikawa, Japonya \\
\hline PTSP & GSJ & $*$ & Nigorikawa, Japonya \\
\hline GEOSIM & Liguori & & San Jacinto-Tizate, Nikaragua \\
\hline GEOSIM & Liguori & & Momotombo, Nikaragua \\
\hline GEOSIM6 & \multicolumn{3}{|c|}{ Tohuku Electric Power Co (Yamanobe) Yanaizu-Nishiyama Japonya } \\
\hline \multirow[t]{28}{*}{ GEOTHERM IIE } & (Barragan R.) & & Cerro Prieto, Meksika \\
\hline & Unocal & * & Awibengkok, Filipinler \\
\hline & UOA Model 4a & & \\
\hline & GeothermEx & $*$ & Miravalles, Kosta Rika \\
\hline & GeothermEx & $*$ & Latera, Italya \\
\hline & GeothermEx & $*$ & Hakkoda, Japonya \\
\hline & GeothermEx & $*$ & Kokubu, Japonya \\
\hline & GeothermEx & $*$ & Minami Aizu, Japonya \\
\hline & GeothermEx & $*$ & Niseko, Japonya \\
\hline & GeothermEx & $*$ & Otake, Japonya \\
\hline & GeothermEx & $*$ & Takigama, Japonya \\
\hline & GeothermEx & $*$ & Yanaizu-Nishiyama, Japonya \\
\hline & GeothermEx & $*$ & MacBan, Filipinler \\
\hline & GeothermEx & $*$ & Tiwi, Filipinler \\
\hline & GeothermEx & $*$ & Boewawe, ABD \\
\hline & GeothermEx & $*$ & Coso Hot Springs, ABD \\
\hline & GeothermEx & $*$ & Desert Peak, ABD \\
\hline & GeothermEx & $*$ & Dixie Valley, ABD \\
\hline & GeothermEx & $*$ & East Mesa, ABD \\
\hline & GeothermEx & $*$ & Heber, ABD \\
\hline & GeothermEx & $*$ & Long Valley, ABD \\
\hline & GeothermEx & $*$ & Puna, ABD \\
\hline & GeothermEx & $*$ & Roosevelt Hot Spr., ABD \\
\hline & GeothermEx & $*$ & Salton Sea, ABD \\
\hline & GeothermEx & $*$ & Soda Lake, ABD \\
\hline & GeothermEx & $*$ & South Brawley, ABD \\
\hline & GeothermEx & $*$ & Steambot Springs, ABD \\
\hline & GeothermEx & $*$ & Stillwater, ABD \\
\hline
\end{tabular}

Not: * işaretli kaynaklara ulaşılamamış olup bilgiler O’Sullivan vd. (2001) den alınmıştır. 
Karahanoğlu

Çizelge 2. TOUGH Programı hakkında (Finsterle vd., 2012).

NAPL $=$ susuz akışkan fazı, NCG = kondanse olmayan gaz, and VOC $=$ uçucu organik bileşik.

Table 2. Overview of TOUGH simulators (Finsterle et al., 2012).

$N A P L=$ non-aqueous phase liquid, $N C G=$ noncondensible gas, and VOC = volatile organic compound .

\begin{tabular}{|c|c|c|c|}
\hline $\begin{array}{l}\text { Simulator } \\
\text { Simülatör }\end{array}$ & $\begin{array}{l}\text { Fazlar, Bileșenler ve Prosesler } \\
\text { Phases, Components, and Processes }\end{array}$ & $\begin{array}{l}\text { Kullanım durumu } \\
\text { Released }\end{array}$ & $\begin{array}{l}\text { Kaynaklar } \\
\text { Key references }\end{array}$ \\
\hline MULKOM & $\begin{array}{l}\text { Newton ve Newton özelliği olmayan, çok fazlı ve } \\
\text { çok bileşenli akışkanlar için ve isı değişkenli ortam } \\
\text { için geliştirilen araştırma kodu } \\
\text { Research code for nonisothermal multiphase, } \\
\text { multicomponent flows of Newtonian and non- } \\
\text { Newtonian fluids }\end{array}$ & $\begin{array}{l}\text { Kullanıma kapalı } \\
\text { No public release }\end{array}$ & Pruess (1983) \\
\hline TOUGH & $\begin{array}{l}\text { Sıvı ve gaz fazında 1sı değişkenli su ve hava akımı } \\
\text { Nonisothermal flow of water and air in aqueous } \\
\text { and gaseous phase }\end{array}$ & 1987 & Pruess (1987) \\
\hline TOUGH2 & $\begin{array}{l}\text { Sıvı ve gaz fazında 1sı değişkenli su ve NCG } \\
\text { (kondanse olmayan gaz) akımı } \\
\text { Nonisothermal flow of water and NCG in aqueous } \\
\text { and gaseous phase }\end{array}$ & 1991 & Pruess (1991) \\
\hline iTOUGH & $\begin{array}{l}\text { TOUGH için ters modelleme } \\
\text { Inverse modeling for TOUGH }\end{array}$ & $\begin{array}{l}\text { Kullanıma kapalı } \\
\text { No public release }\end{array}$ & $\begin{array}{l}\text { Finsterle (1992) } \\
\text { Finsterle vd } 2012 \text { den } \\
\text { alınt1 }\end{array}$ \\
\hline $\mathrm{T} 2 \mathrm{VOC}$ & $\begin{array}{l}\text { Sıv1, gaz ve NAPL fazında 1sı değişkenli su, hava } \\
\text { ve VOC (Uçucu organik bileşen) akımı } \\
\text { Nonisothermal flow of water, air, and VOCs } \\
\text { in aqueous, gaseous, and NAPL phase for } \\
\text { environmental applications }\end{array}$ & 1995 & Falta vd (1995) \\
\hline iTOUGH2 & $\begin{array}{l}\text { TOUGH2 (Pruess, 1991) için ters modelleme, } \\
\text { hassasiyet analizi ve belirsizlik yayılımı analizi ve } \\
\text { ayrica EOS (durum denklemi) modülleri } \\
\text { Inverse modeling, sensitivity analysis, and } \\
\text { uncertainty propagation analysis for TOUGH2 } \\
\text { (Pruess, 1991) and additional EOS modules }\end{array}$ & 1997 & $\begin{array}{l}\text { Finsterle } \\
(1997 a, 1997 b, 1997 c) \\
\text { Finsterle vd } 2012 \text { den } \\
\text { alıntı }\end{array}$ \\
\hline TOUGH2 V2 & $\begin{array}{l}\text { Is1 değişkenli çok fazlı ve çok bileşenli akım } \\
\text { Nonisothermal multiphase, multicomponent flow }\end{array}$ & 1999 & Pruess vd (1999) \\
\hline TMVOC & $\begin{array}{l}\text { Sıvı, gaz ve NAPL fazında 1sı değişkenli su, hava, } \\
\text { çoklu VOC ve NCG akımı } \\
\text { Nonisothermal flow of water, air, multiple VOCs, } \\
\text { and NCGs in aqueous, gaseous, and NAPL phase }\end{array}$ & 2002 & $\begin{array}{l}\text { Pruess ve Batistelli } \\
(2002)\end{array}$ \\
\hline
\end{tabular}


TOUGHREACT Isı değişkenli çok fazlı akım ve reaktif taşınım 2004

(denge ve kinetik mineral disolüsyonu ve presipitasyonu, kimyasal aktif gazlar, sıvı içi ve emme reaksiyonu kinetiği ve biyobozunma)

Nonisothermal multiphase flow and reactive transport including equilibrium and kinetic mineral dissolution and precipitation, chemically active gases, intra-aqueous and sorption reaction kinetics, and biodegradation

TOUGH+

TOUGH2 simülatörünün geliştirilmiş ve genişletilmiş versiyonu, özellikle hidrat içeren jeolojik ortam simülasyonu için Re-engineered and expanded version of TOUGH2 simulator, specifically for the simulation of hydrate-bearing geologic media

TOUGH-FLAC Tam etkileşimli çok fazlı akım ve jeomekanik prosesler için araştırma kodu ; bağlantılar TOUGH2 ve FLAC3D (Itasca, 1997 Finsterle vd 2012 den alınt1)

Research code for coupled multiphase flow and thermal-geomechanical processes; links TOUGH2 and FLAC3D (Itasca, 1997 Finsterle vd 2012 den alınt1)

TOUGH-MP TOUGH 2 nin parallel versiyonu Massively parallel version of TOUGH2

$\mathrm{ECO} 2 \mathrm{M}$
Xu ve Pruess (2001);

Xu vd (2004)

Moridis (2003);

Moridis vd (2008)

Kullanıma Kapalı

No public release

Rutqvist vd (2002)
Geliştirilen sayısal modellerin uygulanmasıyla dünyanın değişik bölgelerindeki jeotermal sahaların sayısal modellemesi yapılarak bu sahaların en uygun şekilde geliştirilmeleri ve optimum ve sürdürülebilir işletilmeleri sağlanmaktadır. Bu konuda yakın zamanlarda yapılan çalışmalara örnek olarak şu makaleler gösterilebilir (Jha ve Puppala, 2018; Quiano ve Zarrouk, 2018; Zeng vd., 2017; Ansari vd., 2017; Weijermars vd., 2017; Turalı ve Şimşek, 2017; Mroczec vd., 2016; Ratouis vd., 2016; Farkutdinov vd., 2016; Canet vd., 2015; Blocher vd., 2015; Zang vd., 2015; Llanos vd., 2015; Moridis ve Freeman, 2015; Finsterle vd., 2014; Lei ve Zhu, 2013; Zeng vd., 2013; Sippel vd., 2013; Cherubini vd., 2013; Gunnarsson vd.,
2012; Kuzevic vd., 2011; Suryadarma vd., 2010). Bunun yanında jeotermal sahalardaki değişik hidrodinamik koşulların modellendiği ve sayısal modellerin geliştirilmeleri yönünde yapılan araştırmalar ve rezervuar ve akışkan fiziksel parametrelerinin sayisal modellemeye etkilerini araştıran çalışmalar da sunulmaktadır (Sonney ve Vuataz, 2009; Blocher vd., 2010; Ingebritsen vd., 2010; Kolditz vd., 2010; Pruess, 2011; Eckart vd., 2011; Yeh vd., 2012; Cerminara ve Fasano, 2012; Ganguly ve Kumar, 2012; Geiger vd., 2012; Finsterle vd., 2012; Bromley vd., 2013; $\mathrm{Hu}$ vd., 2013; Carotenuto vd., 2013; Ostermeyer ve Srisupattarawanit, 2013; Fusi vd., 2013; Jiang vd., 2014; Hathorn vd., 2014; Pogacnik vd., 2014; Dou vd., 2014; Jing vd., 2014; Magnusdottir ve 
Finsterle, 2015; Fairs vd., 2015; Chen vd., 2015; Magliocco vd., 2015; Xu vd., 2015; Xing vd., 2015; Gelet vd., 2015; Pan vd., 2015; Pratama ve Saptadji, 2016; Abdelaziz vd., 2016; Cao vd., 2016; Ansari vd., 2017; Soboleva, 2017; Feng vd., 2017; Randi vd., 2017; Wijaya ve Purqon, 2017; Battistelli vd., 2017) .

İlk kez Brown (2000) tarafından ileri sürülen jeotermal sistemlerde akışkan olarak $\mathrm{CO}_{2}$ kullanılması önerisi 2000 li yıllardan itibaren geçirgenliği çok düşük rezervuarlarda ve kızgın kuru kaya tipi jeotermal sistemlerde modelleme çalışmalarında uygulanmaya başlanmıştır. Pruess (2006) konu ile ilgili olarak araştırmaların başlatıldığını ve $\mathrm{CO}_{2}$ akışan ile modelleme çalışmalarının geliştirildiğini aktarmaktadır. Aynı araştırmada $\mathrm{CO}_{2}$ in suya göre farklı termofiziksel parametrelere sahip olduğunu ileri sürmekte ve enerji üretilmesi konusunda $\mathrm{CO}_{2}$ kullanımının çeşitli avantajlar sağladığını belirtmektedir. Ayrıca bunlara ek olarak $\mathrm{CO}_{2}$ akışkan olarak kullanılmasının rezervuarlarda $\mathrm{CO}_{2}$ depolanmasinı (sequestration) sağlayacağı ileri sürülmüştür. $\mathrm{Bu}$ durum diğer araştırmacılar tarafindan da araştırılmış ve $\mathrm{CO}_{2}$ ve su kullanımının avantaj ve dezavantajları ayrıntılı şekilde tartışılmıştır. Yakın zamanda güçlendirilmiş jeotermal sahaların modellenmesi ile ilgili ayrıntılı araştırmalar yapılmıştır (Hayashi vd., 1999; Pruess, 2006; Jiang vd., 2013; Pan vd., 2015;Biagi vd., 2015; Xu vd., 2015; Magliocco vd., 2015; Xing vd., 2015; Zhao vd., 2015; Chen ve Jiang, 2016).

\section{Türkiye'de Yapılan Modelleme Çalışmaları}

Türkiye'de geçmişten itibaren kaplıca amaçlı kullanılan jeotermal sahaların enerji üretmek amaçlı kullanımı 1968 yılında MTA tarafindan Kızıldere'de yapılan jeotermal sondaj1 ile başlamıştır. Kızıldere sahasının geliştirilmesi amaçlı açılan kuyulardan elde edilen yaklaşık $200{ }^{\circ} \mathrm{C}$ sıcaklıktaki akışkan kullanılarak 20 MWe kapasiteli santral ile elektrik üretilmiştir. Yıllar içerisinde MTA tarafindan bulunan jeotermal sahalar kamu sektörü ve özel sektör aracıllğ 1 ile geliştirilerek enerji üretimi, konut 1sıtmacılığı, seracılık ve balneolojik gibi amaçlarla kullanılmaktadır. Bu konuda yapılan araştırmalar özellikle Ege Bölgesinde yoğun bir şekilde devam etmektedir.

Ülkemizde jeotermal sahaların sayısal modellemesi konusundaki ilk çalışma Karahanoğlu tarafından hazırlanan Doktora Tez çalışması ile literatüre kazandırılmıştır (Karahanoğlu 1983; Karahanoğlu vd., 1984). $\mathrm{Bu}$ araştırmada Kızıldere sahasının sayısal modellemesi yapilarak Kızıldere sahasının üretilmesi ile rezervuarda oluşacak basınç ve sıcaklık değişimlerinin neler olacağı yanında rezervuarda gelişebilecek yüzey çökmesi mekanizması da modellenmiştir. Geliştirilen matematiksel modelde jeotermal rezervuarlardaki hidrodinamik (akım), hidrotermal (enerji) ve mekanik (yüzey çökmesi) süreçlerini tanımlayan diferansiyel denklemler, sicak su tipi jeotermal rezervuarlar için birbirleri ile tam etkileşimli (fully coupled) olarak oluşturulmuştur. Sonlu Elemanlar sayısal modeli kullanılarak çözülen bu denklem sistemlerinin oluşturduğu sayisal model Kızıldere sahasına uygulanmış geçmişteki üretim değerleri kullanılarak model kalibre edilmiş ve o yıllarda yapılan üretim sonucu rezervuar sıcaklığında (sıcaklık), basınç (P) değerlerinde ve yüzey çökmesinde (deplasman) üretim sonucu meydana gelebilecek değişimler tahmin edilmiştir.

Gök vd. (2005) Balçova Narlıdere'deki sıv1 baskın bir jeotermal sahanın üç boyutlu sayısal modellemesini TOUGH2 simulasyon kodu kullanarak yapmıştır. Bu sayısal model jeolojik, 
jeofiziksel, jeokimyasal verilerin yanısıra sondaj ve kuyu testlerinden gelen verileri de içermektedir. Doğal durum modellemesi başlangıçta kuyularda ölçülen sıcaklık ve basınç verileriyle uyum sağlanarak yapılmıştır. Doğal durum modellemesi daha sonra kuyulardaki bütün üretim ve enjeksiyon verilerini (akış oranı, basınç, sıcaklık değerlerinin zaman ile değişimi) yakalayabilmek için kalibre edilmiştir. Model kalibre edildikten sonra toplam rezervuar performansinı ve ayrı ayrı kuyu performansinı belirlemek üzere 20 yıllık tekrar çalıştırılmış ve bu 20 y1llık süreçte kuyudibi basıncı ve sıcaklığ1 cinsinden üretimin sürdürülebilir olduğu gözlemlenmiştir. Buna ek olarak iki yeni kuyu açılması halinde ve aynı enjeksiyon oranlarıla devam edilirse sistemin bir 20 y1l daha sürdürülebilir olduğu belirlenmiştir.

Özkaya (2007) yüksek lisans tez çalışmasında SUTRA programı ile Kızıldere sahasının sayısal modelini çalışmış ve geliştirdiği sayısal modeli geçmişteki üretim değerlerini kullanarak jeotermal sahaya kalibre etmiştir. Daha sonra sahada mevcut tüm kuyuları kullanarak değişik üretim ve enjeksiyon senaryolarına karş1lık zaman içerisinde olabilecek sicaklık ve basınç değişimlerini tahmin etmiş ve farklı enjeksiyon ve üretim koşullarının etkilerini ortaya koymuştur.

Can ve Budak (2008) hazırladıkları iki boyutlu sayısal modeli Kizıldere sahasina uygulamışlar ve rezervuarı gözenekli ortam olarak değerlendirmişler ve akışkan üretimini tek bir kuyudan yapılıyor şeklinde varsaymışlardır. 1984-1998 yılları arasındaki üretim değerlerini kullanarak modeli kalibre etmişler ve gözlenen değerlerle uyumlu sonuçlar aldıklarını ve sonuç olarak mevcut kuyuların 20 MWe elektrik üretmek için yeterli olmayacağını ifade etmişlerdir.
Magri vd. (2010) yaptıkları çalışmada Narlıdere-Balçova derin jeotermal sistemindeki jeotermal akışkan dinamiği ve enerji taşınımını tam etkileşimli olarak modellemeyi araştırmışlardır. Yapılan araştırmada doğal beslenim miktarlarının, jeotermal sistemdeki faylar boyunca gelişebilecek akışkan dinamiği süreçlerinin değişik jeotermal rezervuar oluşumlarına neden olabileceği ve deniz suyu girişimi riskinin mümkün olabildiği, dikey yöndeki fayların bu konuda etkili olduğu ve derinlerde gelişip yükselen sıcak suların dengesiz olduğu ve dolayısı ile sıcaklıklarda salınımlara neden olduğu ifade edilmiştir. Yapılan bu araştırma sonunda Seferihisar-Balçova derin jeotermal sistemindeki modelleme çalışmalarının kalitatif olarak rezervuarda gelişen değişik akım koşullarını modellediği belirtilmiştir.

Günay ve Karahanoğlu (2015) Edremit jeotermal sahasının sayısal modelini yapmış ve sahada yapılan değişik üretim senaryolarının rezervuara etkisini araştırmıştır. Edremit jeotermal sahasının kavramsal modeli kullanılarak zamana bağl1, iki boyutlu kesit sonlu elemanlar modeli oluşturulmuş ve 1 lik ve sicak iki farklı seviyeden oluşan jeotermal sistemin doğal durum kalibrasyonunu takiben değişik üretim ve enjeksiyon koşullarında rezervuarların davranışı araştırılmıştır.

Yoğurtcuoğlu (2016) Edremit jeotermal sahasının üç boyutlu sonlu elemanlar entegre modelini gerçekleştirmiştir. Rezervuarda hidrolik eğim değerlerinin bulunması için iki boyutlu alansal model yapılmış ve sonraki aşamada bu değerler üç boyutlu sonlu elemanlar modeli ile entegre edilerek sayısal model çalışması gerçekleştirilmiştir. Sayısal modelin sahaya kalibre edilmesinden sonra farklı senaryolar üretilerek jeotermal sistemin sürdürülebilir üretim ve enjeksiyon koşullarının etkisi irdelenmiştir. 
Karahanoğlu

Turalı ve Şimşek (2017) yaptıkları araştırmada TOUGH2 simülatörü kullanarak Sorgunda bulunan jeotermal sistemi modellemiştir. Bu çalışmada uygun sınır koşulları ve fiziksel parametreler kullanılarak kavramsal model hazırlanmış ve üretim öncesi kuyularda statik sicaklık değerleri kullanılarak doğal rezervuar koşulları modellenmiştir. Modellenen ve gözlenen sıcaklık değerleri arasında iyi bir uyum elde edildikten sonra 2007-2014 aras1 üretim/enjeksiyon ve entalpi üretim değerleri kullanılarak sayısal modelin tarihsel kalibrasyonu gerçekleştirilmiş ve gözlenen su seviyeleri ve sıcaklık değerlerinin hesaplananlarla iyi uyum sağladığ görülmüştür.

2007 yılında çıkartılan Jeotermal Kaynaklar ve Doğal Mineralli Sular Kanunu ile jeotermal sahaların özel sektörler tarafından geliştirilmeleri ve işletilmeleri konusu gündeme gelmiştir. $\mathrm{Bu}$ durum yüksek sıcaklığa sahip jeotermal sahaların enerji amaçlı olarak üretilmelerini sağlamış ve dolayısı ile mevcut sahaların geliştirilerek enerji santrallerinin kurulmalarını gerekli kılmıştır. Halen birçok sahada (Kızıldere, Germencik gibi) enerji santralleri kurulmuştur ve jeotermal rezervuarlar işletilerek elektrik üretilmektedir. Jeotermal sahaların işletim haklarının özel sektörlere devredilmeleri neticesinde mevcut rezervuarların kapasiteleri yeni kuyular açılarak geliştirilmiş ve yüksek kapasiteli santraller kurularak elektrik enerjisi üretimi sağlanmıştır.

İşletim haklarının özel sektörlere devredilmeleri mevcut jeotermal sahaların geliştirilmeleri konusunda olumlu adımların atılmasını sağlamıştır ancak bu sahalarda yapılan sayısal modelleme çalışmaları konusundaki bilgi paylaşımı asgari düzeye indirilmiştir. Ruhsat sahibi firmaların ticari kaygı ile modelleme çalışmaları hakkındaki bilgileri kamuoyu ile paylaşmamaları durumu ortaya çıkmıştır. Bunun sonucu olarak ülkemizdeki sayısal modelleme çalışmaları hakkında yayımlanan bilgiler kamuya açık sahalarda yapılan araştırmalarla ve Jeotermal Kaynaklar ve Doğal Mineralli Sular Kanunu çıkarılmadan önceki dönemlerde yapılan çalışmalarla sınırlı kalmıştır.

Jeotermal enerji santrallerinin kurulmas öncesinde yapılması gerekli olan sayısal modelleme çalışmalarının genellikle yurt dışı kaynaklı profesyonel firmalarca yapıldığ 1 tahmin edilmektedir. Bu durum ise jeotermal enerji kapasitesi açısından dünyanın önde gelen ülkeleri arasında yer alan ülkemizde jeotermal sahaların sayısal modellemesi konusundaki araştırmaların yapılabilmesinin son derece kısıtlı olduğu gerçeğini ortaya koymaktadır.

\section{TEMEL PRENSIPLER VE MODEL DENKLEMLERI}

Jeotermal rezervuarlarda akışkan hareketini ve 1s1 yay1lımın1 kontrol eden hidrotermal ve hidrodinamik mekanizmalar etkilidir. Bu nedenle jeotermal rezervuarların sayısalmodellenebilmesi için bu iki mekanizmanın rezervuar ortamında ve rezervuar sınırlarında matematiksel terimlerle ifade edilmeleri gerekmektedir.

\section{Matematiksel Model Denklemleri}

Jeotermal rezervuarlarda akışkan hareketi ve enerji taşınımı mekanizmaları matematiksel olarak kısmi diferansiyel denklemler kullanılarak ifade edilmektedir. Denklemler oluşturulurken kütlenin ve enerjinin korunumu prensipleri ve devamlılık kuramı esas alınmaktadır. $\mathrm{Bu}$ çerçevede oluşturulan denklemlerin ayrıntılarına Karahanoğlu vd. (1984) çalışmasında ulaşılabilir. Model denklemlerinin son halleri aşağıda verilmektedir: 


\section{Akım Denklemi}

Jeotermal rezervuarlarda akışkan hareketinin matematiksel olarak ifade edilmesi için kütlenin korunması ve akımın devamlılığ ilkeleri ve Darcy yasası esas alınmıştır. Bu ilkeler doğrultusunda hazırlanan diferansiyel denklem tek fazlı akışkan için aşağıda verilmektedir: olduğu ve mekan koordinatları ile etkilendiği görülmektedir. Ayrıca bu denklem içinde sıcaklık değişkenin de yer aldığı ve böylelikle tek denklem sistemi içinde iki adet bilinmeyen değişken bulunduğu izlenmektedir.

$$
\begin{array}{r}
\frac{\partial}{\partial X_{i}} k_{i j} \frac{\partial}{\partial X_{j}}\left[\frac{P}{Y^{f}}+H\right]-\Phi \beta^{f} \frac{\partial T}{\partial t}+\frac{\Phi}{K_{B}^{f}} \frac{\partial P}{\partial t} \\
+(1-\Phi) \beta^{s} \frac{\partial T}{\partial t}+\frac{\partial Q f}{\partial t}=0
\end{array}
$$

$\mathrm{Bu}$ denklemde $\mathrm{P}$ basınç değişkenini ve $\mathrm{T}$ ise sıcaklık değişkenini göstermektedir. Ayrıca $\mathrm{k}$ ortamın permeabilitesini, $\mathrm{H}$ belirli bir seviyeden yüksekliği, $\Phi$ gözenekliliği, ' $\mathrm{Y}^{\mathrm{f}}$ akışkan birim ağırlığını, $\beta^{\mathrm{f}}$ ve $\beta^{\mathrm{s}}$ akışkan ve katı kısmın 1sıl genleşme katsayılarını, Q terimi ise birim zamandaki akım miktarını ifade etmektedir. Denklem dikkatli incelendiğinde, fiziksel parametrelerin sabit oldukları bir tarafa bırakılırsa, basınç değişkeninin zamana bağlı

\section{Enerji Denklemi}

Gözenekli ortamda 1S1 hareketi Enerjinin korunumu prensibi kullanılarak aşağıdaki kısmi diferansiyel denklemle ifade edilmektedir (Karahanoğlu vd, 1984). Konvektif ve kondüktif 1S1 taşınım terimlerini içeren bu denklem yazılırken akışkan yoğunlunun sıcaklık ve basınç değişkenlerine bağlı olduğu varsayılmış diğer katsayılar sabit olarak alınmıştır.

$$
\begin{gathered}
{\left[(1-\Phi) \rho_{s} c_{s}+\Phi \rho_{f} c_{f}-\Phi \rho_{f} c_{f} \beta^{f} T\right] \frac{\partial T}{\partial t}-\frac{\partial}{\partial X_{i}} K_{i j} \frac{\partial T}{\partial X_{j}}} \\
+\frac{\Phi \rho_{f} C_{f}}{K_{B}^{f}} T \frac{\partial P}{\partial t}+\Phi \rho_{f} C_{f} v_{i}^{a} \frac{\partial T}{\partial X_{i}}-\frac{\partial Q_{h}}{\partial t}=0
\end{gathered}
$$


Karahanoğlu

Denklemde yer alan $\rho_{\mathrm{s}}, \mathrm{c}_{\mathrm{s}}$ ve $\rho_{\mathrm{f}}, \mathrm{c}_{\mathrm{f}}$ terimleri katı ve akışkanların yoğunluklarını ve birim 1S1 kapasitelerini göstermekete ve $K_{\text {ij }}$ ise $1 \mathrm{~s} 1$ kondüktivite matrisini ifade etmektedir. (1) ve (2) nolu denklemler dikkatlice incelendiğinde akım denkleminde sıcaklık değişkenine bağlı terim, enerji denkleminde ise basınç değişkenine bağlı terim yer almakta ve bu durum her iki denklemin birbirleri ile tam etkileşimli (fully coupled) olduklarını göstermektedir. Diğer taraftan akım denkleminde olduğu gibi bu denklem sisteminde de iki adet bilinmeyen değişken ( $P$ ve $T$ ) bulunmaktadır ve böylelikle iki denklem sistemi ile iki bilinmeyen değişken eşitliği elde edilmiş olmaktadır.

Jeotermal rezervuarlarda akışkan ve 1s1 taşınımını ifade eden bu denklemlerin yanında sınır koşullarının da matematiksel olarak yazılması gerekmektedir. Bu konudan hareketle sınırda akışkan hareketinin devamlılığının sağlanabilmesi

$$
-n_{i} k_{i j} \frac{\partial}{\partial X_{j}}\left(\frac{P}{Y^{f}}+H\right)-\frac{\partial q_{f}}{\partial t}=0
$$

ifadesi ile sağlanmakta ve $\partial \mathrm{q}_{\mathrm{f}} / \partial \mathrm{t}$ ise sınırda birim alandaki akışkan boşalım hızını göstermektedir. Sabit sinır değerleri olarak $\mathrm{P}=$ $\mathrm{P}^{\mathrm{b}}$ ve $\mathrm{T}=\mathrm{T}^{\mathrm{b}}$ sınırda bilinen basınç ve sıcaklık değerleri kullanılmaktadır. Sınırdaki kondüktif 1S1 akımının devamlılığ

$$
-n_{i} K_{i j} \frac{\partial T}{\partial x_{j}}-\frac{\partial q_{h}}{\partial t}=0
$$

ifadesi ile gerçekleştirilmekte ve yine sınırdaki konvektif 1s1 akımının devamlılığ ise

$$
-n i K_{i j} \frac{\partial T}{\partial X_{j}}-h^{T}\left(T-T_{f}\right)=0
$$

şeklinde yazılmaktadır ve denklemdeki $\mathrm{h}^{\mathrm{T}}$ terimi konvektif ısı taşınımı katsayısını, $\mathrm{T}_{\mathrm{f}}$ ise referans sınır sıcaklık değerini ve $n_{i}$ sinıra dik birim vektörü göstermektedir.

Sınır koşullarının belirlenmesi ile (1) ve (2) nolu denklemlerle jeotermal rezervuarlarda tek fazlı akışkan akımı ile enerji taşınımının matematiksel olarak modellenmesi gerçekleştirilmiş olmaktadır. Sayısal modellemenin yapılabilmesi için yukarda yazılan denklem sistemlerinin sayısal olarak çözülmesi ve böylece bilinmeyen değişkenlerin zaman ve mekan boyutlarındaki dağılımlarının bulunması gerekmektedir.

\section{Sayısal Model}

Önceki kısımda yazılan (1) ve (2) nolu kısmi diferansiyel denklemlerin (3), (4) ve (5) nolu sınır koşullarıyla birlikte çözülmesi için Sonlu Elemanlar veya Sonlu Farklar yöntemi uygulanarak denklemler Lineer Cebirsel Denklem sistemlerine dönüştürülmektedir. Matriks yöntemi ile ifade edildiğinde sonlu elemanlarda elemanların düğüm sayısı, sonlu farklarda ise grid noktaları sayısı kadar denklem ve bir o kadar bilinmeyenden oluşan denklemler elde edilmektedir. $\mathrm{Bu}$ denklemlerin uygun sınır koşulları ve rezervuarı en iyi şekilde temsil eden fiziksel parametreler kullanılarak çözülmesi ile bilinmeyen değişkenlerin (basınç, sicaklık) rezervuar ortamında ve zaman içindeki dağılımları bulunmaktadır. Elde edilen bu çözüm değerleri gözlenen değerlerle karşılaştırılarak gerçek çözüm değerlerinin elde edilmesi sağlanmaktadır. Gözlenen değerlerle hesaplanan değerler arasındaki farklar kalibrasyon denilen yöntemler kullanılarak minimum seviyeye indirilmektedir. 


$$
\left\{\begin{array}{cc}
\mathrm{K}_{\mathrm{ij}} \\
\end{array}\right]=\left\{\begin{array}{c}
\mathrm{P}_{\mathrm{i}} \\
\mathrm{T}_{\mathrm{i}} \\
\cdot \\
\cdot \\
\cdot \\
\mathrm{P}_{\mathrm{m}} \\
\mathrm{T}_{\mathrm{m}}
\end{array}\right\}=\left\{\begin{array}{c} 
\\
\mathrm{R}_{\mathrm{i}} \\
\\
\\
{ }_{1 \times m}
\end{array}\right\}
$$

$\mathrm{Bu}$ denklem sisteminde $\mathrm{K}$ matrisi denklemdeki sabit katsayılardan oluşmakta ve sağ taraftaki $R$ vektörü ise denklemin bilinenlerini içermektedir. Denklem sisteminin bilinmeyenleri olan $\mathrm{P}$ ve $\mathrm{T}$ değişkenleri ilgili bilinmeyenler vektörü içinde bulunmaktadır. Denklem sistemi bu şekli ile bilinmeyenlerin rezervuar içindeki dağılımlarını vermektedir ve zaman içindeki değişimleri elde etmek için aşağıdaki denklem sisteminin çözülmesi gerekmektedir.
$\mathrm{Bu}$ denklemde $\Delta \mathrm{t}$ zaman aralığını göstermekte ve bilinmeyen değişkenlerin ( $P$, $\mathrm{T})$ yeni zaman $(\mathrm{t}+\Delta \mathrm{t})$ dilimindeki değerlerini hesaplamak için bilinmeyen değişkenlerin eski zaman $(\mathrm{t})$ dilimindeki değerleri kullanılmaktadır. Sonlu elemanlar yönteminde her bir eleman için yazılan bu denklemler eleman düğüm noktaları için birleştirilerek global denklemler elde edilmekte ve denklem sistemlerinin sayısal olarak çözülebilmeleri için sınır koşullarının

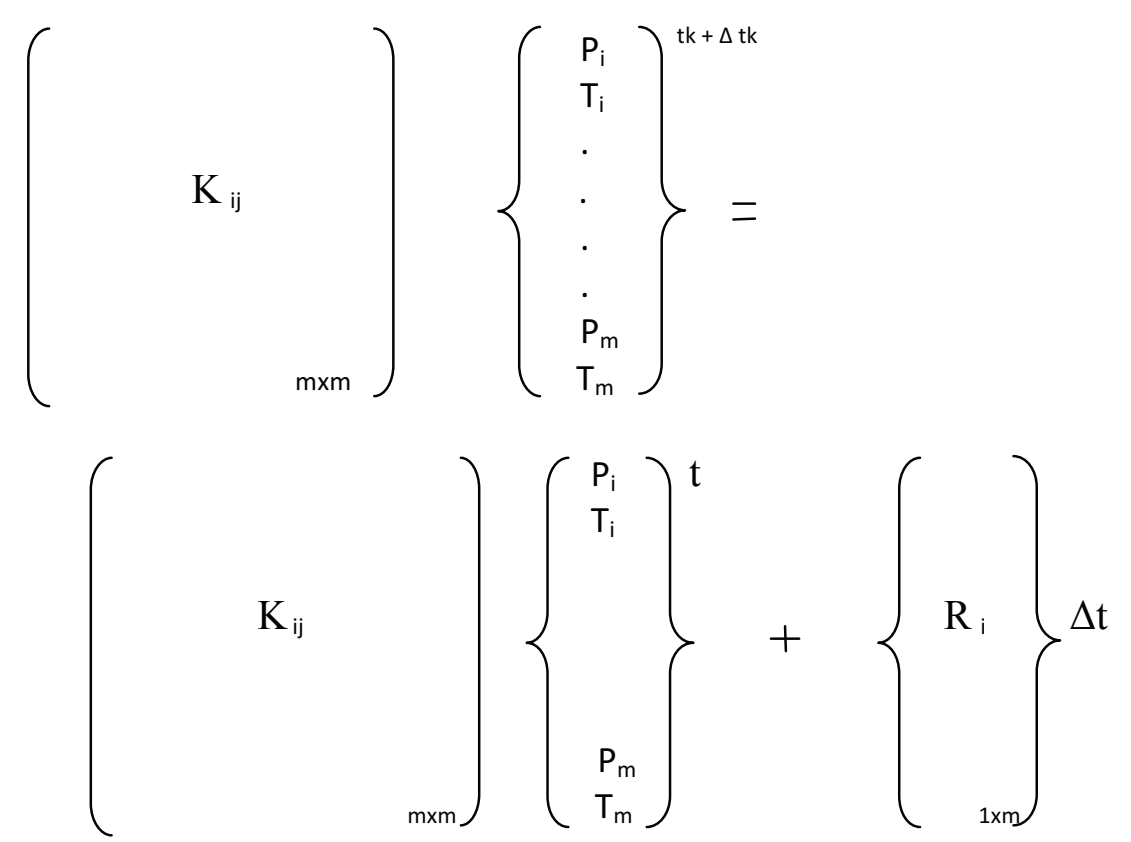

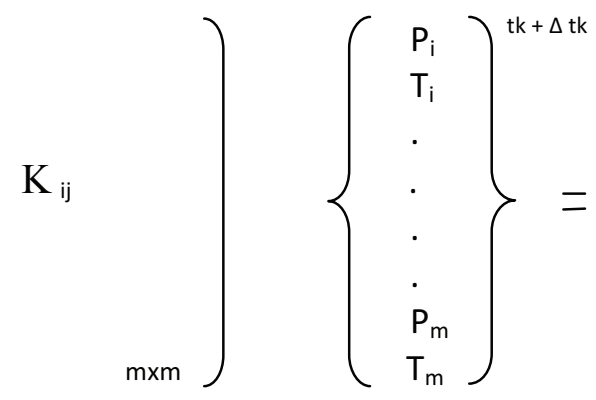


Karahanoğlu

tanımlanması yani rezervuar sınırlarındaki hidrodinamik ve hidrotermal süreçlerin matematiksel olarak ifade edilmeleri gereklidir. Dinamik sayısal model oluşturabilmek için ayrıca başlangıç koşullarının belirlenmesi gerekmektedir. Sınır koşulları ve başlangıç koşulları belirlenirken bilinmeyen değişkenlerin (P basınç, $\mathrm{T}$ sıcaklık) rezervuar sınırlarındaki hidrodinamik ve hidrotermal davranışlarının sayısal modele tanıtılmaları şarttır. Jeotermal rezervuarın başlangıç koşulları olarak rezervuarın doğal denge durumu (rezervuarın işletmeye açılmadan önceki durumu) değerlendirilmekte ve basınç ve sıcaklık değerlerinin rezervuardaki dağılımlarıtahmin edilmektedir. Sınır koşullarının belirlenmesi ise rezervuar sınırlarında basınç ve sıcaklık bilinmeyenleri değerlerinin veya bu değişkenlerin türevlerinin matematiksel olarak ifade edilmeleri ile gerçekleştirilmektedir.

Başlangıç ve sınır koşullarının belirlenmesi ile denklem sistemleri sayısal çözüme hazır duruma getirilmekte ve bu denklemlerin çözülmesi sonucu değişkenlerin değişik zaman dilimleri içinde jeotermal rezervuar ortamındaki dağılımları elde edilmektedir.

Genel olarak bu çerçevede gerçekleştirilen ve sayısal modellemede kullanılarak sahalara uygulanan simülatörler zaman içerisinde geliştirilerek jeotermal rezervuarlarda gelişen her türlü doğal süreçlerin simülasyonu yapılmaktadır. Ancak bu tür simülatörleri oluşturan kaynak programlara ulaşım mümkün değildir. Dolayısı ile simülatörün öngördüğü standart rezervuar koşulları dışındaki süreçlerin modellenmesinde sorunlar oluşabilmektedir. Ayrıca kaynak programa ulaşım olmaması nedeniyle sayısal modelleme konusunda yer bilimcilerimizin eğitilmeleri ciddi anlamda kısıtlanmaktadır.

\section{GENEL DEĞERLENDİRMELER VE SONUÇLAR}

Jeotermal rezervuarların sayısal yöntemler kullanılarak modellenmesi ve jeotermal sistemlerin üretimi ile rezervuarlarda basınç ve sıcaklık değişimlerinin bilimsel olarak takip edilebilmesi 1970 li y1llardan beri yapılan araştırmalarla gerçekleştirilebilmektedir. 1960 lı y1llarda Jeotermal sistemlerdeki doğal mekanizmaların matematiksel olarak ifade edilmeleri şeklinde başlayan araştırmalar 1970 lerde bilgisayar teknolojisinde gözlenen hızlı gelişmelerle ivme kazanmış ve sayısal yöntemler kullanılarak rezervuarların modellenmesi ve dolayısı ile sistemlerin bilimsel olarak ve sürdürülebilir bir şekilde üretilmelerine imkan sağlanmıştır. Önceleri iki boyutlu ve tek fazlı akışkanlar için hazırlanan sayısal modeller zaman içerisinde çok fazlı (sıvı, gaz) akışkanların (sıcak $\mathrm{su}$, buhar, hava, $\mathrm{CO}_{2}$ ) üç boyutlu rezervuarlardaki hareketini modelleyebilecek duruma gelmiş ve akışkan hareketi ve enerji taşınımı mekanizmaları ötesinde yüzey çökmesi, kimyasal reaksiyonlar, CO2 depolanmas1/gömülmesi, çeşitli jeomekanik prosesler, biyodejenerasyon ve benzer mekanizmaların değerlendirildiği simülatörler geliştirilmiştir. İlk yıllarda sabit fiziksel parametreler kullanılarak hazırlanan sayisal modeller zaman içerisinde basinç ve sıcaklık değişkenlerine bağlı parametrelerin kullanıldığı modellere dönüştürülmüştür.

Sayısal modelleme konusunda yapılan çalışmaların zaman içerisindeki evrimine bakıldığında ilk yıllarda ticari olmayan ve kullanıma açık kodların kullanıldığı ve model çalışmalarının bilimsel ortamlarda paylaşıldığ 1 görülmektedir. Ancak zaman içerisinde yapılan çalışmalarda program çabalarının paylaşılması yerine bilinen programların değişik sahalara uygulandığ 1 ve karşılaşılan sorunların tartışıldığ 
toplantılar yapılarak malum programların iletilen sorunları çözme yönünde geliştirildikleri anlaşılmaktadır.

Sayısal modelleme çalışmalarının önemli bir aşamasını jeotermal rezervuarların kavramsal modelinin hazırlanması ve sistemin fiziksel özelliklerinin bilgisayar ortamına aktarılması oluşturmaktadır. Matematiksel denklemler kullanılarak ifade edilen ısı taşınımı ve akışkan hareketi mekanizmalarının modeli yapılan sahaya uygulanabilmesi ilgili sahanın tüm özelliklerinin simülatöre tanıtılması ile mümkün olmaktadır. Denklemlerde yer alan çeşitli parametrelerin arazi ortamındaki değerlerinin belirlenmesi ve bu değerlerin dağılımlarının bulunması hazırlanan sayısal modelin başarısı açısından son derece önemlidir. Diğer taraftan rezervuarın sinır ve başlangıç koşullarının yani ilgili mekanizmaların sınırlardaki davranışlarının matematiksel olarak ifade edilmesi ve rezervuarın üretime açılmadan önceki doğal durumunun (basınç ve sıcaklık dağılımlarının) gerçekçi olarak belirlenmesi model başarısını etkileyen çok önemli bir husustur.

Doğal ortamda çeşitli etmenler altında ve uzun yıllar içinde oluşan jeotermal sistemlerdeki karmaşıklığın, ve jeotermal sistemlerin yapısal unsurlarının ve tüm fiziksel özelliklerinin kavramsal modeller yardımı ile sayısal modellere aktarılması söz konusu fiziksel parametrelerin rezervuar ortamını en iyi şekilde temsil etmesi ile gerçekleşmektedir. $\mathrm{Bu}$ durum bahsedilen fiziksel parametrelerin, ve denklemlerde bulunan katsayıların belirlenmesinde çok titiz araştırmaların (arazi ve laboratuvar çalışmaları) yapılması gerekliliğini ortaya koymaktadır. Benzer şekilde jeotermal sistemlerin sinır ve başlangıç koşullarının arazi koşullarını temsil edecek türde belirlenmesi yapılacak modelleme çalışmalarının başarısını en üst düzeyde etkilemektedir. Böylelikle doğadaki jeotermal sistem/rezervuar bilgisayar ortamına aktarılarak sayısal model oluşturulmakta ve model sistem/ rezervuar simülatör kullanılarak değişik üretim ve enjeksiyon senaryolarının sisteme yapacağ etkiler araștırılabilmektedir.

Sayısal modelleme çalışmalarının zaman içerisindeki gelişimi ve bu konuda dünya genelinde yapılanlar gözden geçirildiğinde simülasyon çalışmalarının çok başarılı olduğu ve bu bağlamdajeotermalrezervuarların dahaverimli ve sürdürülebilir şekilde değerlendirildikleri anlaşılmaktadır. Hemen hemen her türlü karmaşık mekanizmaların modellenebilir olmasının yanında çok farklı jeolojik yapıların kavramsal model şeklinde tanımlanabildiği sayısal modeller oluşturulabilmektedir.

Son yıllarda yapılan araştırmalar ülkemizin tektonik yapısının jeotermal sahaların oluşumu için çok elverişli olduğu gerçeğini ortaya koymaktadır. Ege bölgesinde mevcut yüksek entalpili jeotermal sahaların elektrik enerjisi elde etmek için üretildikleri ve bu sahaların geliştirilmeleri yönünde araştırmaların devam ettiği bilinmektedir. Jeotermal enerji açısından belli bir zenginliğe sahip ülkemizin bu kaynaklarının verimli bir şekilde kullanılabilmesi için bu sahaların bilgisayar teknolojisi kullanılarak değerlendirilmesi gereklidir. $\mathrm{Bu}$ konuda elde edilen teknoloji ve bilgi birikiminden yararlanma anlamında yerbilimcilerin eğitilmesinin önemli olduğu vurgulanmalıdır.

Ülkemizde bulunan jeotermal sahaların sayısal modellemesi konusunda yapilan bilimsel araştırma ve çalışmalar çok az sayıda olup genelde bilinen programların ilgili sahalara uygulaması şeklinde gerçekleşmektedir. Sayısal modelleme çalışmalarında saha verisi zenginliğinin önemi bilinmekle beraber son 
yıllarda yapılan işletim politikaları nedeniyle veri paylaşımının çok kısıtlandığ 1 ve bu durumun söz konusu araştırmaları olumsuz yönde etkilediği bir gerçektir.

\section{KATKI BELIRTME}

$\mathrm{Bu}$ makalenin hakemler tarafindan değerlendirilmesi sırasında verdikleri değerli katkılar için Sayın Prof. Dr. Şakir Şimşek’e ve ismini bilmediğim Hakem'e teşekkürlerimi iletiyorum. Yaptıkları çok titiz ve ayrıntılı incelemeleri ve yapıcı eleştirileri makalenin üstün kaliteye ulaşmasını sağlamıştır. Ayrıca makalenin hazırlanması aşamasında Çizelge 1 deki emeği için Emre Günay’a teşekkür ediyorum.

\section{KAYNAKLAR}

Abdelaziz, R., Komori, F.S., Carreno, M.N.P., 2016. Multiphase thermal-fluid flow through geothermal, International Scientific Conference - Environmental and Climate Technologies, Conect 2015, Riga, Latvia, Oct 14-15, 2015, Energy Procedia, 95, 22-28.

Aboustit, B. L., Advani, S.H., Lee, J.K., 1985. Variational principles and finite element simulations for thermo-elastic consolidation. International Journal for Numerical and Analytical Methods in Geomechanics, 9, 45-69.

Absar, K.V., Bajpai,, I.P., Sinha, A.K., Ashwini, K., 1996. Reservuar modeling of Puga geothermal system, Iadakh, Jammua and Kashmir. Geological Survey, India, 69-74.

Amistoso, E.A., Aquino, B.G., Aunzo, Z.P., Jordan, O.T., Sta. Ana, F.X.M., Bovardsson. G.S., Daughty, C., 1993. Reservoir analysis of the Palinpinon geothermal field, Negros Oriental, Philippines, Geothermics, 22 (5/6), 555-574.

Ansari, E., Hughes, R., White, C.D., 2017. Statistical modeling of geopressured geothermal reservoirs. Computers and Geosciences, 103, 36-50.
Antics, M., 2000. Computer simulation of geothermal reservoirs in the Pannonian Basin, Eastern Europe. Proceedings World Geothermal Congress, Kyushu-Tohuku, Japan, May 28-June 10 2000, 2497-2502.

Antics, M.A., 1998. Computer modeling of an over pressured medium enthalpy geothermal reservoir located in deep sedimentary basin. Proceedings of the 23 rd Workshop on Geothermal Reservoir Engineering, Stanford University, Stanford, California, 26-28 January 1998, 362-367.

Antics, M.A., 1997. Computer simulation of Oradea geothermal reservoir Proceedings of the 22nd Workshop on Geothermal Reservoir Engineering, Stanford University, Stanford, California, 27-29 January 1997, 491-495.

Antunez, E.U., Menzies, A.J., Sanyal, S.K., 1991. Simulating a challenging water dominated geothermal system: the Cerro Prieto field, Baja, California, Mexico. Proceedings of $16^{\text {th }}$ Workshop on Geothermal Reservoir Engineering, Stanford University, Stanford, California, 23-25 January 1991, 183-191.

Antunez, E.U., Sanyal, S.K., Carella, R., Guidi, A., 1990. Quantitative verification of the hydrogeological model of the Mufete geothermal field, Campania, Italy. Transactions geothermal Resources Council 14, 1263-1270.

Antunez, E.U., Bodvarsson, G.S., Walters, M.A.,1994. Numerical-Simulation Study of the Northwest Geysers Geothermal-Field, a Case-Study of the Coldwater Creek Steamfield. Geothermics, 23, 2, 127-141.

Arellano, V., Garcia, A., Barragan, R., Izquierdo, G., Aragon, A., Nieva, D., 2003. An updated conceptual model of the Los Humeros geothermal reservoir (Mexico). Journal of Volcanology and Geothermal Research, 127, 67-88.

Arias, A., Dini, I., Cassini, M., Fiordelisi, A., Perticone, I., Dell'Aiuto, P., 2010. Geoscientific feature update of the Larderello-Travale geothermal system (Italy) for a regional numerical modeling. In: Proc World geothermal congress 2010, Bali, Indonesia. 
Arihara, N., Yoshida, H., Hanano, M., Ikeuchi, K., 1995. A simulation study on hydrothermal system of the Kakkonda geothermal field. Proceedings World Geothermal Congress'95, Florence, 18-31 May 1995, 1715-1720.

Arnorsson, S., 1995. Geothermal systems in Iceland: structure and conceptual models-I. High temperature areas. Geothermics, 24, 561-602.

Atmojo, J.P., Itoi, R., Tanaka, T., Fukuda, M., Sudarman, S., Widiyarso, A., 2000. Modeling studies of Sudayak geothermal reservoir Northern Sumatra, Indonesia. Proceedings of World Geothermal Congress, Kyushu-Tohuku, Japan, May 28-June 102000 2037-2042.

Aunzo, Z., Steingrimsson, B., Bodvarsson, G.S., Escobar, C., Quintanilla, A., 1989. Modeling studies of the Ahuachapan geothermal field, El Salvador. Proceedings of the 14 th workshop on geothermal reservoir engineering report, Stanford University, Stanford, California, 26-28 January 1989, 287-295.

Axelsson, G., Bjornsson, G., 1993. Detailed three dimensional modeling of the Btn hydrothermal system in N-Iceland. Proceedings of the 18 th workshop on geothermal reservoir engineering, Stanford, California (USA), 26-28 January 1993, 159-166.

Barelli, A., Ceccarelli, A., Dini, I., Fiordelisi, A., Giorgi, N., Lovari, F., 2010a. A review of the Mt. Amiata geothermal system, Italy. In: Proc World geothermal congress 2010, Bali, Indonesia.

Barelli, A., Cei, M., Lovari, F., Romagnoli, P., 2010b. Numerical modeling for the LarderelloTravale geothermal system, Italy. In: Proc World geothermal congress 2010, Bali, Indonesia.

Barmin, A.A., Kondrashov, A.V., 2000. Two-front mathematical model of water injection into a steam-saturated geothermal reservoir, Fluid Dynamics, 35, 3, 399.

Bataille, A., Genthon, P., Rabinowicz, M., Fritz, B., 2006. Modeling the coupling between free and forced convection in a vertical permeable slot: Implications for the heat production of an Enhanced Geothermal System. Geothermics, 35, 5-6, 654-683.
Battistelli, A., Calore, C., Pruess, K., 1997. The simulator TOUGH2/EWASG for modelling geothermal reservoirs with brines and noncondensible gas, Geothermics, 26, 4, 437-464.

Battistelli, A., Swenson, D., Alcott, A., 2017. Improved PetraSim-TOUGH2 capabilities for the simulation of Geothermal reservoirs, In: Proc of $42^{\text {nd }}$ workshop on geothermal reservoir engineering, Stanford, California (USA).

Battistelli, A., Yiheyis, A., Calore, C., Ferragina, C., Abatneh, W., 2002. Reservoir engineering assessment of Dubti geothermal field, Northern Tendaho Rift, Ethiopia, Geothermics, 31, 381406.

Battistelli, A., Yiheyis, A., Calore, C., Ferragina, C., Abathneh, W., 1998. Tendaho geothermal project (Ethiopia): Reservoir engineering studies in the Dubti area. Proceedings of the World Renewabla Energy Congress V, Florence 1998, 2741-2745.

Battistelli, A., Calore, C., Rossi, R., Wu, F., 1992. Reservoir engineering study of Nagqu geothermal field (Tibet autonomous region, PRC). Presented at the High Temperature Geothermal Resources Workshop, Lhasa, 9-16 August 1992.

Bear, J., Çorapçığlu, M.Y., 1981. A mathematicalmodel for consolidation in a thermoelastic aquifer due to hot water injection or pumping, water resources research, 17, 3, 723-736.

Bertani, R., Capetti, G., 1995. Numerical simulation of the Monteverdi zone (western border of the Larderello geothermal field). Proceedings World Geothermal Congress'95, Florence, 18-31 May 1995, 1735-1740.

Biagi, J., Agarwal, R., Zhang, Z.M., 2015. Simulation and optimization of enhanced geothermal systems using $\mathrm{CO} 2$ as a working fluid, Energy, $86,627-637$.

Bjornsson, G., 1999. Predicting future performance of a shallow steam zone in the Svartsengi geothermal field, Iceland. Proceedings of the $24^{\text {th }}$ Workshop on Geothermal Reservoir Engineering, Stanford University, Stanford, California, 25-27 January 1999 116-122. 
Karahanoğlu

Blocher, M.G., Zimmermann, G., Moeck, I., Brandt, W., Hasanzadegan, A., Magri, F., 2010. 3D numerical modeling of hydrothermal processes during the lifetime of a deep geothermal reservoir, Geofluids, 10, 406-421.

Blocher, G., Cacace, M., Reinsch, T., Watanabe, N., 2015. Evaluation of three exploitation concepts for a deep geothermal system in the North Germain Basin, Computers and Geosciences, 82, 120-129.

Bloomfield, K.K., Moore, J.N., Sperry, T.L., 1998. Cove-Fort Sulphurdale reservoir numerical simulation. Transactions Geothermal Resources Council, 22, 149-152.

Boardman, S., Ali Khan, M., Artunez, E., 1996. TOUGH'/PC Application simulation project for Heber geothermal field, California, a progress report. Proceedings of the $21^{\text {st }}$ workshop on geothermal reservoir engineering, Stanford, California (USA).

Bodvarsson, G.S., 1988. Model predictions of the Svartgensi reservoir, Iceland, water Resources Research, 24 (10), 1740-1746.

Bodvarsson, G.S., Bjornsson, J., Gunnarsson, A., Gunnlaugsson, E., Sigurdsson, O., Stefansson, V., Steingrimsson, B., 1990a. The Nesjavellir geothermal field, Iceland: 1. Field characteristics and development of a three-dimensional numerical model. Journal of Geothermal Science and Technology, 2 (3), 189-228.

Bodvarsson, G.S., Pruess, K., Haukwa, C., Ojiambo, S.B., 1990b. Evaluation of model predictions for the Olkaria East Geothermal Field, Kenya, Geothermics, 19, (5), 399-414.

Bodvarsson, G.S., Pruess, K., Lippmann, M., Bjornsson, S., 1981. Improved Energy Recovery From Geothermal Reservoirs, 56 $6^{\text {th }}$ Annual Technical Conference and Exhibition of the Society of Petroleum Engineers of AIME, San Antonio, Texas, USA.

Bodvarsson, G.S., Pruess, K., Lippmann, M., Bjornsson, S., 1982. Improved energy recovery from geothermal reservoirs. Journal of Petroleum Engineers Journal, 1920-1928.
Bodvarsson, G.S., Pruess, K., Stefansson, V., Bjornsson, S., Ojiambo, S.B., 1987a. East Olkaria Geothermal Field, Kenya: 1. History match with production and pressure decline data, Journal of Geophysical Research: Solid Earth,vol 92, issue B1, 521-539.

Bodvarsson, G.S., Pruess, K., Stefansson, V., Bjornsson, S., Ojiambo, S.B., 1987b. East Olkaria Geothermal Field, Kenya: 2. Predictions of well performance and reservoir depletion Journal of Geophysical Research, 92 (B1), 541554.

Bodvarsson, G.S., Pruess, K., Lippmann, M.J., 1986, Modeling of geothermal systems, Journal of Petroleum Technology, 1007-1021.

Bromley, C., Brockbank, K., Glynn-Morris, T., Rosenberg, M., Pender, M., O’Sullivan, M., Currie, S., 2013. Geothermal subsidence study at Wairakei-Tauhara, New Zealand Proceedings of the Institution of Civil Engineers-Geotechnical Engineering, 166, 2, 211-223.

Brown, D., 2000. A hot dry rock geothermal energy concept utilizing $\mathrm{CO} 2$ instead of water. In: Proceedings of $25^{\text {th }}$ workshop on geothermal reservoir engineering, Stanford CA, USA: Jan 2000. P. 233-8.

Brownell, D.H., Garg, S.K., Pritchett, J.W., 1977. Governing equations for geothermal reservoirs, Water Resources Research, 13, 929-934.

Bujakowski, W., Tomaszewska, B., Miecznik, M., 2016. The Podhale geothermal reservoir simulation for long-term sustainable production, Renewable Energy, 99, 420-430.

Burnell, J.G., 1992. Modeling mass, energy and chloride flows in the Rotorua geothermal system. Geothermics, 21(1/2), 261-280.

Butler, S.J., Sanyal, S.K., Henneberger, R.C., Klein, C.W., Gutierrez, H., de Leon, J.S., 2000. Numerical modeling of the Cerro Prieto geothermal field, Mexico. In: Proc of world geothermal congress, Kyushu, Japan.

Canet, C., Trillaud, F., Prol-Ledesma, R.M., GonzalezHernandez, G., Pelaez, B., Hernandez-Cruz, B., Sanchez-Cordova, M.M., 2015. Thermal history of the Acoculco geothermal system, eastern 
Mexico: Insights from numerical modeling and radiocarbon dating, Journal of Volcanology and Geothermal Research, 305, 56-62.

Can, I., Budak, A., 2008. Finite element modeling of the Kizildere geothermal system, Turkey, Energy Sources Part A-Recovery Utilization and Environmental Effects, 30, 12, 1098-1107.

Cao, W.J., Huang, W.B., Jiang, F.M., 2016. Numerical study on variable thermophysical properties of heat transfer fluid affecting EGS heat extraction. International Journal of Heat and Mass Transfer, 92, 1205-1217.

Carlino, S., Toriano, A., Di Guiseppe, M.G., Tramelli, A., Troise, C., Somma, R., De Natale, G. 2016. Exploitation of geothermal energy in active volcanic areas: A numerical modeling applied to high temperature Mofete Geothermal field, at Campi Flegrei caldera (Southern Italy). Renewable Energy, 87, 1, 54-66.

Carotenuto, A., Massarotti, N., Mauro, A., 2013. A new methodology for numerical simulation of geothermal down-hole heat exchangers. Applied Thermal Engineering, 48, 225-236.

Carradori, G., Peano, A., Voss, C.I., 1981. The geophase model for finite element simulation of multiphase geothermal reservoirs, in R.W. Lewis and Schrefler (Ed.) Numerical Methods in Thermal Problems, Proc. $2^{\text {nd }}$ Int Conference in Venice, Pineridge Press, Swansea, 315-325.

Cerminara, M., Fasano, A., 2012. Modeling the dynamics of a geothermal reservoir fed by gravity driven flow through overstanding saturated rocks. Journal of Volcanology and Geothermal Research, 233-234, 37.

Chen, F., Falta, R.W., Murdoch, L.C., 2015. Numerical Analysis of Thermal Remediation in 3D FieldScale Fractured Geologic Media, Groundwater, 4, 572-587.

Chen, J.L., Jiang, F.M., 2016. A numerical study of EGS heat extraction process based on a thermal non-equilibrium model for heat transfer in subsurface porous heat reservoir, Heat and Mass Transfer, 52, 2, 255-267.
Cherubini, Y., Cacace, M., Scheck-Wenderoth, M., Moeck, I., Lewerenz, B., 2013. Controls on the deep thermal field: implications from 3-D numerical simulations for the geothermal research site Gro Schonebeck, Environmental Earth Sciences, 70, 8, 3610-3642.

Croucher, A.E., O'Sullivan, M.J., 2008. Application of the computer code TOUGH2 to the simulation of supercritical conditions in geothermal systems. Geothermics, 37, 622-634.

Driesner, T., Geiger, S., 2007. Numerical simulation of multiphase fluid flow in hydrothermal systems, Fluid-Fluid Interactions, Reviews in Mineralogy \& Geochemistry, Ed:Liebscher, A., Heinrich, C.A., $65,187-212$.

Dou, B., Gao, H., Zhou, G., Ren, L., 2014. Numerical simulation of heat transfer from hot dry rock to water flowing through a circulation fracture, $3 \mathrm{rd}$ International Conference on Materials Science and Engineering (ICMSE 2014), Jiujiang, Peoples R China, Jan 24-26, 2014, Material Science and Advanced Technologies in Manufacturing, Book Series: Advanced Materials Research, 852, 831834.

Eckart, M., Kories, H., Ruterkamp, P., Kaul, V., Bems, C., 2011. Flow-and Heat-Transport- simulation with an optimized discretization of the geological structure model, International-Mine-WaterAssociation Congress on Mine Water - Managing the Challenges, Aachen, Germany, Sep 04-11, 2011, Ed:Rude, T.R., Freund, A. Wolkersdorfer, C., 167-170.

Emoricha, E.B., Omagbon, J.B., Malate, R.C.M., 2010. Three dimensional numerical modeling of Mindanao geothermal production field, Phillipines.In: Proc of the $35^{\text {th }}$ Workshop on Geothermal reservoir engineering, Stanford, California (USA).

Ertekin T., 1978. Numerical simulation of the compaction-subsidence phenomena in a reservoir for two phase non-isothermal flow. PhD Thesis, The Pennsylvania State University, 227p.

Esberto, M.B., Sarmiento, Z.F., 1999. Numerical modeling of the Mt. Apo geothermal reservoir. In. Proc. of the $24^{\text {th }}$ workshop on reservoir engineering, Stanford, California (USA). 
Fabbri, P., Trevisani, S., 2005. Spatial distribution of temperature in the low-temperature geothermal Euganean field (NE Italy): a simulated annealing approach, Geothermics, 34, 5, 617-631.

Fairs, T.H., Younger, P.L., Parkin, G., 2015. Parsimonious numerical modelling of deep geothermal reservoirs, Proceedings of The Institution of Civil Engineers-Energy, 168, 4, 218-228.

Falta, R.W., Pruess, K., Finsterle, S., 1995. T2VOC User's Guide. Report LBLN-36400, Lawrence Berkeley Laboratory, Berkeley CA, 165 pp.

Farkhutdinov, A., Goblet, P., de Fouquet, C., Cherkasov, S., 2016. A case study of the modeling of a hydrothermal reservoir: Khankala deposit of geothermal waters. Geothermics, 59, 56-66, Part: A.

Farkhutdinov, A., Goblet, P., De Fouquet, C., Ismagilov, R., Farkhutdinov, I., Cherkasov, S., 2015. The Use of Computer Modelling to Forecast the Sustainability in the Development of Geothermal waters Resource: Khankala Deposit Example. International Journal of Renewable Energy Research, 5, 4, 1062-1068.

Faust, C.R., Mercer, J.W., 1975. Mathematical modeling of geothermal systems. Proc $2^{\text {nd }} U N$ Symposium on the Development and Use of Geothermal Resources, San Francisco, 3, 16331642.

Faust, C.R., Mercer, J.W., 1979a. Geothermal Reservoir Simulation.1. Mathematical-Models For Liquid-Dominated and Vapor-Dominated Hydrothermal Systems, Water Resources Research, 15, 1, 23-30.

Faust, C.R., Mercer, J.W., 1979b. Geothermal Reservoir Simulation. 2. Numerical Solution Techniques For Liquid-Dominated and VaporDominated Hydrothermal Systems, Water Resources Research, 15, 1, 31-46.

Fendek, M., 2000. Reservoir modeling study of Galanta area. Proceedings World Geothermal Congress Kyushu-Tohuku, Japan, , May 28June 10 2000, 2555-2560.

Fendekova, M., Fendek, M., 2000. Evaluation of
Horna-Nitra geothermal reservoir (central part of Slovak Republic). Proceedings World Geothermal Congress Kyushu-Tohuku, Japan, , May 28- June 10 2000, 2561-2565.

Feng, G., Tianfu.G., Jiang, Z.J., Bellani, S., 2017. Geothermal assessment of the Pisa plain, Italy: Coupled thermal and hydraulic modeling, Renewable Energy, 111, 416-427.

Finsterle, S., Kowalsky, MB., Pruess, K., 2012. TOUGH model use, calibration and validation, Transactions of the ASABE 55 (4), 1275-1290.

Finsterle, S., Sonnenthal, E.L., Spycher, N., 2014. Advances in subsurface modeling using the TOUGH suite of simulators, Computers \& Geosciences, 65, 2-12, SI.

Finsterle, S., 1993. iTOUGH2 user's guide version 2.2, Lawrence Berkeley Laboratory, University of California.

Franco, A., Vaccaro, M., 2014. Numerical simulation of geothermal reservoirs for the sustainable design of energy plants: A review, Renewable \& Sustainable Energy Reviews, 30, 987- 1002.

Fusi, L., Rosso, F., Ceseri, M., Borsi, I., Speranza, A., 2013. Weak formulation for a two-phase nonlinear flow in an undeformable porous medium, Meccanica, 48, 1, 57-70.

Ganguly, S., Kumar, M.S.M., 2012. Geothermal reservoirs - A brief review, Journal of the Geological Society of India, 79, 6, 589-602.

Garg, S.K., Pritchett, J.W., Brownell Jr., D.H., 1975. Transport of mass and energy in porous media, Proc $2^{\text {nd }}$ UN Symposium on the Development and Use of Geothermal Resources, San Francisco, 3, 1651-1656.

Geiger, S., Schmid, K.S., Zaretskiy, Y., 2012. Mathematical analysis and numerical simulation of multi-phase multi-component flow in heterogeneous porous media, Current Opinion in Colloid \& Interface Science, 17, 3, 147-155.

Gelet, R.M., Loret, B., Khalili, N., 2015. The significance of local thermal non-equilibrium in simulations of enhanced geothermal recovery, Conference: Computer Methods and Recent 
Advances in Geomechanics, Kyoto, Japan, Sep 22-25, 2014, Ed:Oka, F., Murakami, A., Uzuoka, R., Kimoto, S., 1713-1718.

Gök, I.M., Sarak, H., Onur, M., Serpen, U., Satman, A., 2005. Numerical modeling of the Balçova Narlıdere geothermal field, Turkey, Proceedings World Geothermal Congress 2005, Antalya, Turkey.

Guerrero-Martinez, F.J., Verma, S.P., 2013. Three dimensional temperature simulation from cooling of two magma chambers in the Las Tres Virgenes geothermal field, Baja, California Sur, Mexico, Energy, 52, 110-118.

Gunnarsson, G., Arnaldsson, A., Oddsdottir, A.L., 2012. Model Simulations of the Hengill Area, Southwestern Iceland, Transport of Unsaturated Groundwater and Heat (TOUGH) Symposium, Berkeley, CA, Sep 14-16, 2009, Transport in Porous Media, 90, 1, 3-22.

Gupta, M., Rao, G., Narain, H., 1974. Geothermal investigations in the Puga Valley hot spring region Ladakh, India, Geophysical Research Bulletin, 12, 119-136.

Gupta, M.L., Sharma, S., Singh, S., Drolia, R., 1979, Geophysical exploration and assessment of power potential of Puga Geothermal field. Geoviews, 6, 1-4.

Günay, E., Karahanoğlu, N., 2015. Finite element simulation of the Edremit geothermal field, Proc of 40th workshop on geothermal reservoir engineering, Stanford University, p:1-10.

Hadgu, T., Zimmerman, R.W., Bovardson, G.S., 1995. Coupled Reservoir-Wellbore Simulation of Geothermal Reservoir Behavior, Geothermics, 24, 2, 145-166.

Hanano, M., 1998. A simple model of a two-layered high-temperature liquid-dominated geothermal as a part of a large-scale hydrothermal convection system, Transport in Porous Media, 33, 1-2, 3-27.

Hanano, M., 1992. Simulation Study of the Matsukawa Geothermal Reservoir - Natural State and its Response to Exploitation, Journal of Energy Resources Technology-Transactions of the ASME, 114, 4, 309-314.
Hathorn, D., Wu, Y.S., Chen, Z.Z., 2014. TOUGH2PETSc: A Parallel Solver for TOUGH2, 2014. 15th International Conference on Parallel And Distributed Computing, Applications and Technologies (PDCAT 2014), Hong Kong, PEOPLES R CHINA, Dec 09-11, 2014, 174179.

Hayashi, K., Willis-Richards, J., Hopkirk, R.J., Niibori, Y., 1999. Numerical models of HDR geothermal reservoirs - a review of current thinking and progress, Geothermics, 28, 4-5, 507-518.

Hu, B., 1995. Reservoir simulation of the Yangbajian geothermal field in Tibet, China. Proceedings World Geothermal Congress'95, 18-31 May 1995 Florence, 1691-1695.

Hu, L.T., Winterfeld, P.H., Fakcharoenphol, P., Wu, Y.S., 2013. A novel fully-coupled flow and geomechanics model in enhanced geothermal reservoirs, Journal of Petroleum Science and Engineering, 107, 1-11.

Ingebritsen, S.E., Sorey, M.L., 1985. Quantitative analysis of the Lassen hydrothermal system, north-central California. Water Resourses research, 21 (6), 853-868.

Ingebritsen, S.E., Geiger, S., Hurwitz, S., Driesner, T., 2010. Numerical Simulation of Magmatic Hydrothermal Systems, Reviews of Geophysics, 48.

Ishido, T., Tosha, T., 1998. Feasibility study of reservoir monitoring using repeat self potential measurements Transactions Geothermal Resources Council 22, 171-177.

Itoi, R., Kumamoto, Y., Tanaka, T., Takayama, J., 2010. History matching simulation of the Ogiri geothermal field, Japan. In: Proc World geothermal congress 2010, Bali, Indonesia.

Jha, S.K., Puppala, H., 2018. Conceptual modeling and characterization of Puga geothermal reservoir, Ladakh, India, Geothermics, 72, 326-337.

Jiang, F.M., Luo, L., Chen, J.L., 2013. A novel threedimensional transient model for subsurface heat exchange in enhanced geothermal systems, International Communications in Heat and Mass Transfer, 41, 57-62. 
Karahanoğlu

Jiang, F.M., Chen, J.L., Huang, W.B., Luo, L., 2014. A three-dimensional transient model for EGS subsurface thermo-hydraulic process, Energy, $72,300-310$.

Jing, Y.N., Jing, Z.Z., Willis-Richards, J., Hashida, T., 2014. A simple 3-D thermoplastic model for assessment of the long-term performance of the Hijiori deep geothermal reservoir, Journal of Volcanology and Geothermal Research, 269, 1422.

Karahanoğlu, N., Doyuran, V., Akkaş, N., 1984. Finite Element Simulation of Hot-Water type Geothermal Reservoirs, Journal of Volcanology and Geothermal Research, 23, 3-4, 357-382.

Karahanoğlu, N., 1983. Finite Element Simulation of Hot-Water type Geothermal Reservoirs, PhD Thesis, Middle East Technical University, Ankara Turkey.

Karrech, A., Beltaief, O., Vincec, R., Poulet, T., Regenauer-Lieb, K., 2015. Coupling of thermalhydraulic-mechanical processes for geothermal reservoir modelling, Journal of Earth Science, $26,1,47-52$.

Kiryukhin, A.V., 1996. Modeling studies: The Dachny geothermal reservoir, Kamchatka, Russia, Geothermics, 25, 1, 63-90.

Kiryukhin, A.V., Asaulova, N.P., Finsterle, S., 2008. Inverse modeling and forecasting for the exploitation of the Pauzhetsky geothermal field, Kamchatka, Russia. Geothermics, 37, 540-562.

Kiryukhin, A.V., Asaulova, N.P., Finsterle, S., Rychkova, T.V., Obora, N.V., 2006. Modeling the Pauzhetsky geothermal field, Kamchatka, Russia, using iTOUGH2. In: Proc TOUGH Symposium, Lawrence Berkeley National Laboratory, California (USA).

Kiryukhin, A.V., Asaulova, N.P., Manukhin, Y.F., Rychkova, T.V., Sugrobov, V.M., 2010. Using numerical modeling for assessing the recoverable reserves of a geothermal steam field: The Pauzhetka geothermal field, Journal of Volcanology and Seismology, 4, 52-71.

Kissling, W.M., Brown, K.L., O’Sullivan, M.J., White, S.P., Bullivant, D.P., 1996. Modeling chloride and $\mathrm{CO} 2$ chemistry in the Wairakei geothermal reservoir. Geothermics, 25, 285-305.

Kolditz, O., Clauser, C., 1998. Numerical simulation of flow and heat transfer in fractured crystalline rocks: Application to the hot dry rock site in Rosemanowes (UK). Geothermics, 27, 1-23.

Kolditz, O., Blöcher, M. G., Clauser, C., Diersch, H.-J. G., Kohl, T., Kühn, M., McDermott, C. I., Wang, W., Watanabe, N., Zimmermann, G. and Bruel, D., 2010. Geothermal Reservoir Simulation, in Geothermal Energy Systems: Exploration, Development, and Utilization (ed E. Huenges), Wiley-VCH Verlag GmbH \& Co. KGaA, Weinheim, Germany.

Köhn, M., Stöfen, H., 2005. A reactive flow model of the geothermal reservoir Waiwera, New Zealand, Hydrogeology Journal, 13, 4, 606-626.

Kumamoto, Y., Itoi, R., Tanak, T., Hazama, Y., 2009. Modeling and numerical analysis of the two-phase geothermal reservoir at Ogiri, Kyushi, Japan. In: Proc of the $34^{\text {th }}$ Workshop on Geothermal reservoir engineering, Stanford, California (USA).

Kuzevic, S., Kuzevicova, Z., Hojdova, M., Pusztai, A., 2011. A Computer-Based Simulation of Geothermal Energy Utilization in Conditions of Slovak Republic, $11^{\text {th }}$ International Multidisciplinary Scientific Geoconference (SGEM 2011), VOL III, Albena, Bulgaria, Jun 20-25, 2011.

Lasseter, T.J., 1976. Numerical-Simulation of Heat and Mass-Transfer in Multi-dimensional 2-Phase Geothermal Reservoirs, Mechanical Engineering, 98, 5, 104-104.

Lei, H.Y., Zhu, J.L., 2013. Numerical modeling of exploitation and reinjection of the Guantao geothermal reservoir in Tanggu District, Tianjin, China, Geothermics, 48, 60-68.

Lewis, R.W., Karahanoğlu, N., 1981. Simulation of Subsidence in Geothermal Reservoirs, Numerical Methods in Thermal Problems, 2, 326-335.

Lewis, R.W., Roberts, P.J., Schrefler, B.A., 1989. Finite element modeling of two phase heat and fluid flow through deforming porous media, Transport in Porous Media, 4, 319-334. 
Lipmann, M.J., Narasimhan, T.N., Witherspoon, P.A., 1976. Numerical simulation of reservoir compaction in liquid dominated geothermal systems. Proc. 2nd Int Symposium on Land Subsidence, Anaheim, Ca., 157-166.

Llanos, E.M., Zarrouk, S.J., Hogarth, R.A., 2015. Numerical model of the Habanero geothermal reservoir, Australia, Geothermics, 53, 308-319.

Magliocco, M.J., Glaser, S.D., Kneafsey, T.J., 2015. Laboratory and Numerical Studies of Heat Extraction from Hot Porous Media by Means of Supercritical CO2, TOUGH Symposium, Berkeley, CA,Sep 17-19, 2012, Transport in Porous Media, 108, 1, 85-104.

Magnusdottir, L., Finsterle, S., 2015. An iTOUGH2 equation-of-state module for modeling supercritical conditions in geothermal reservoirs, Geothermics, 57, 8-17.

Magri, F., Akar, T., Gemici, U., Pekdeger, A., 2010. Deep geothermal groundwater flow in the Seferihisar-Balcova area, Turkey: results from transient numerical simulations of coupled fluid flow and heat transport processes, Geofluids, 10, 3, 388-405.

Mannington, W., O’Sullivan, M., Bullivant, D., 2000. An air/water model of the Wairakei-Tauhara geothermal system. In: Proc of world geothermal congress, Kyushu, Japan.

Mannington, W., O’Sullivan, M., Bullivant, D., 2004. Computer modeling of the Wairakei-Tauhara geothermal system, New Zealand. Geothermics, 33, 4, 401-419.

Mc.Guinnes, M.J., White, S.P., Young, R.M., Izhisaki, H., Ikeuchi, K., Yoshida, Y., 1995. A model of the Kakkonda geothermal reservoir. Geothermics, 24, 1-48.

Menzies, A.J., Granados, E.E., Sanyal, S.K., Merida-I, L., Caicedo-A., A., 1991.Numerical modeling of the initial state and matching well test data from the Zunil geothermal field Guatemala. Proceedings of the 16 th Workshop on Geothermal Reservoir Engineering, Stanford University, Stanford, California, 23-25 January 1991, 193-201.
Menzies, A.J., Pham, M., 1995. A field-wide numerical simulation model of The Geysers geothermal field, California, Proceedings World Geothermal Congress'95, Florence, 18-31 May 1995, 1697-1702.

Mercer, J.W., Faust, C.R., 1979. Geothermal Reservoir Simulation.3. Application of Liquid-Dominated and Vapor-Dominated Hydrothermal Modeling Techniques to Wairakei, New-Zealand, Water Resources Research, 15, 3, 653-671.

Mercer, J.W., Faust, C.R., and Pinder, G.F.,1974. Geothermal reservoir simulation. Conference on research for the Development of Geothermal Energy Resources, Pasadena, California, 256267.

Morgan, K., Lewis, R.W., White, I.R., 1980. The mechanisms of ground surface subsidence above compacting multi phase reservoirs and their analysis by the finite element method, Applied Mathematical Modelling, 4, 217-234.

Moridis, G.J., 2003. Numerical studies of gas production from methane hydrates. Society of Petroleum Engineers Journal 32(8), 359-370.

Moridis, G.J., Freeman, C.M., 2015. The RealGas and RealGasH2O options of the TOUGH plus code for the simulation of coupled fluid and heat flow in tight/shale gas systems. Computers \& Geosciences, 65, 56-71, SI.

Moridis, G.J., Kowalsky, M.B., Pruess, K., 2008. TOUGH+HYDRATE v1.0 User's Manuel: A code for the Simulation of System Behaviour in Hydrate-Bearing Geologic Media. Report LBNL-149E, Lawrence Berkeley national Laboratory, CA, 279 pp.

Morris, C.W., Campbell, D.A., 1981. Geothermal Reservoir Energy recovery - A 3-Dimensional Simulation Study of the East Mesa Field. Journal of Petroleum Technology, 33, 4, 735-742.

Mottaghy, D., Pechnig, R., Vogt, C., 2011.The geothermal project Den Haag: 3D numerical models for temperature prediction and reservoir simulation. Geothermics, 40, 3, 199-210.

Mroczec, E.K., Milicich, S.D., Bixley, P.F., Supelveda, F., Bertrand, E.A., Soengkono, S., Rae, A.J., 
2016. Ohaaki geothermal system: Refinement of a conceptual reservoir model. Geothermics, 59, 311-324.

Nakanishi, S., Abe, M., Todaka, N., Yamada, M., Sierra, J.L., Gingins, M.O., Mass, L.C., Pedro, G.E., 1995. Copahue geothermal system, Argentina- study of a vapor dominated reservoir. Proceedings World Geothermal Congress '95, Florence, 18-31 May 1995, 1167-1172.

Nakanishi S., Iwai, N., 2000. Reservoir simulation study of the Onikobe geothermal field, Japan. Proceedings World Geothermal Congress, Kyushu-Tohuku Japan, May 28-June 10, 2000, 2159-2164.

Nguyen, V.V., Pinder, G.F., 1983. Geothermal Reservoir Simulation using, Non-Equilibrium Thermodynamics. Society of Petroleum Engineers Journal, 23, 4, 613-622.

Noorollahi, Y., Itoi, R., 2011. Production capacity estimation by reservoir numerical simulation of northwest (NW) Sabalan geothermal field, Iran, Energy, 36, 7, 4552-4569.

Ostermeyer, G.P., Srisupattarawanit, T., 2013. MultiScale Simulation of Heat and Flow in Geothermal Reservoirs, Oil Gas-European Magazine, 39, 1, 40-42.

O'Sullivan, M.J., 1985. Geothermal Reservoir Simulation. International Journal of Energy Research, 9, 3, 319-332.

O'Sullivan, M., Barnett, B., Razali, M., 1990. Numerical simulation of the Kamojang geothermal field, Indonesia, Trans Geotherm Resources Counc 1317-1324.

O’Sullivan, M.J., Bullivant, D.P., Follows, S.E., Mannington, W.I., 1998. Modeling of the Wairakei-Tauhara geothermal system. Proceedings of the TOUGH Workshop'98, Berkeley, California, 4-6 May 1998, 1-6.

O’Sullivan, M.J., Pruess, K., Lippmann, M.J., 2001. State of the art of geothermal reservoir simulation. Geothermics, 30, 4, 395-429.

O’Sullivan, M.J., Yeh, A., Mannington, W.I., 2009. A history of numerical modeling of the Wairakei geothermal field. Geothermics, 38, 1, 155-168.
Özkaya M., 2007. Numerical modeling of the Kizıldere Geothermal field. MSc Thesis, Middle East Technical University, Ankara, Turkey.

Pan, L.H., Freifeld, B., Doughty, C., Zakem, S., Sheu, M., Cutright, B., Terrall, T., 2015. Fully coupled wellbore-reservoir modeling of geothermal heat extraction using $\mathrm{CO} 2$ as the working fluid, Geothermics, 53, 100-113.

Parini, M., Cappetti, G., Laudiano, M., Bertani, R., Monterrossa, M., 1995. Reservoir modeling study of the Ahuachapan geothermal field (El Salvador) in the frame of a generation stabilization project. Proceedings World Geothermal Congress '95, Florence, 18-31 May 1995, 1543-1548.

Parini, M., Acuna, J.A., Laudiano, M., 1996. Reinjected water return at Miravalles geothermal reservoir, Costa Rica: numerical modeling and observations. Proceedings of the 21st Workshop on Geothermal Reservoir Engineering, Stanford University, Stanford, California, 22-24 January 1996, pp 127-134.

Pearson, S.C.P., Alcaraz, S.A., Barber, J., 2014. Numerical simulations to assess thermal potential at Tauranga low-temperature geothermal system, New Zealand. Hydrogeology Journal, 22, 1, 163174.

Pham, M., Sanyal, S.K., Menzies, A.J., Naka, T., Takeuchi, R., Iata, S., 1995. Numerical modeling of the high temperature two-phase reservoir at Uenotai geothermal field, Akita prefecture, Japan. Proceedings World Geothermal Congress'95, Florence, 18-31 May 1995, 1703-1707.

Pham, M., Menzies, A.j., Sanyal, S.K., Lima, E., Shimada, K., Juarez, J., Cuevas, A., 1996. Numerical modeling of the high temperature geothermal system of Amatitlan, Guatemala. Transactions Geothermal Resources Council 20, 833-838.

Pogacnik, J., Dempsey, D., Kelkar, S., Podgorney, R., O’Sullivan, M., O'Sullivan, J., 2014. The Effect of Sequential Solution Procedures in the NumericalModeling of Stimulation in Engineered Geothermal Systems, 11th World Congress on Computational Mechanics; 5th European Conference on Computational Mechanics; $6^{\text {th }}$ 
European Conference on Computational Fluid Dynamics, Barcelona, Spain, Jul 20-25, Vols II - IV, Ed:Onate, E; Oliver, X; Huerta, A., 13071318.

Porras, E.A., Tanaka, T., Fujii, H., Itoi, R., 2007. Numerical modeling of the Momotombo geothermal system, Nicaragua. Geothermics, 36, 4, 304-329.

Porras, E.A., Tanaka, T., Fujii, H., Itoi, R., 2005. Numerical modeling of the Momotombo geothermal system, Proceedings World Geothermal Congress 2005, Antalya, Turkey.

Portugal, E., Birkle, P., Tello, E., Tello, M., 2000. Hydrochemical-isotopic and hydrogeological conceptual model of the Las Tres Virgenes geothermal field, Baja, California Sur, Mexico. Journal of Volcanology and Geothermal Research, 101, 223-244.

Pratama, H.B, Saptadji, N.M.,2016. Numerical Simulation for Natural State of Two-Phase Liquid Dominated Geothermal Reservoir with Steam Cap Underlying Brine Reservoir, 5th ITB International Geothermal Workshop (IIGW2016), Bandung, Indonesia, Mar 27-Apr 02, 2016, Book Series: IOP Conference SeriesEarth and Environmental Science, Ed:Saepuloh, A; Suryantini; Wibowo, H; Deon, F; Sianipar, J.Y., 42, Article Number: 012006.

Pritchett, J.W., Garg, S.K., 1995. A modelling study of the Oguni geothermal field, Kyushu, Japan, Proceedings World Geothermal Congress'95, Florence, 18-31 May 1995, pp 1703-1707.

Pritchett, J.W., Garg, S.K., Ariki, K., Kawano, Y., 1991. Numerical simulation of the Sumikawa geothermal field in the natural state. In: Proc of the 16 th workshop on geothermal reservoir engineering, Stanford, California (USA).

Pruess, K., Batistelli, A., 2002. TMVOC A numerical simulator for Three-Phase Non-Isothermal flows of Multicomponent Hydrocarbon mixtures in saturated, unsaturated heterogeneous media. Report LBLN-49375E, Lawrence Berkeley National Laboratory, Berkeley CA, 192 pp.
Pruess, K., 2006. Enhanced geothermal systems (EGS) using $\mathrm{CO}_{2}$ as working fluid- a novel approach for generating renewable energy with simultaneous sequestration of carbon. Geothermics, 35(4), 351-367.

Pruess, K., 1983. Development of the general purpose simulator MULKOM, Annual Report 1982, Earth Sciences Division Report LBL-15500, Lawrence Berkeley Laboratory, Berkeley, CA, $103 \mathrm{pp}$.

Pruess, K., 1991. TOUGH2 A General Purpose Numerical Simulator for Multiphase Fluid and Heat Flow. Report LBL-29400. Lawrence Berkeley Laboratory, Berkeley, CA, 103 pp.

Pruess, K., Oldenburg, C., Moridis, G., 1999. TOUGH2 user's guide version 2.1. Report LBLN-43134, Lawrence Berkeley National Laboratory, Berkeley CA, 204 pp.

Pruess, K., Zerzan, J.M., Schroeder, R.C., Witherspoon, P.A., 1979. Description of the three dimensional two phase simulator SHAFT78 for use in geothermal reservoir studies, paper SPE7699 presented at the SPE Fifth symposium on reservoir simulation, Denver, Jan 31-Feb 2 1979.

Pruess, K., Bodvarsson, G.S., Schroeder, R.C., Witherspoon, P.A., 1982. Model Studies of the Depletion of Two-Phase Geothermal Reservoirs. Society of Petroleum Engineers Journal, 280290.

Pruess, K., 1990. Modeling of geothermal reservoirs: Fundamental processes, computer simulation and field applications. Geothermics, 19, 1, 3.

Pruess, K., 1987. TOUGH Users Guide. Report LBL-20700. Lawrence Berkeley Laboratory, Berkeley, CA, 78 pp.

Pruess, K., 2011. ECO2M: A TOUGH2 fluid property module for Mixtures of Water, $\mathrm{NaCl}$, and $\mathrm{CO}_{2}$. Including Super- and Sub critical Conditions, and Phase change between liquid and gaseous $\mathrm{CO}_{2}$. Report LBLN-4590E, Lawrence Berkeley National Laboratory, Berkeley CA, 83pp.

Pruess, K., 2003. The TOUGH codes - A family of simulation tools for multiphase flow and 
transport processes in permeable media, TOUGH Symposium 2003, La wrence Berkeley National Lab, Berkeley, Ca, May, 2003, Vadose Zone Journal, 3, 3, 738-746.

Quiano, J.J.D., Zarrouk, S.J., 2018. Geothermal resource assessment using experimental design and response surface methods: The Ngatamariki geothermal field, New Zealand, Renewable Energy, 116, 325-334.

Randi, A., Sterpenich, J., Thiery, D., Kervevan, C., Pironon, J., Morlot, C., 2017. Experimental And Numerical Simulation of the Injection of $\mathrm{A} \mathrm{CO}_{2}$ Saturated Solution in a Carbonate Reservoir: Application to the $\mathrm{CO}_{2}$-Dissolved Concept Combining $\mathrm{CO}_{2}$ Geological Storage and Geothermal Heat Recovery, 13th International Conference on Greenhouse Gas Control Technologies, Ghgt-13, Lausanne, Switzerland, Nov 14-18, 2016, Book Series: Energy Procedia,114, Ed:Dixon, T; Laloui, L; Twinning, S. 2942-2956.

Ratouis, T.M.P., O’Sullivan, M.J., O’Sullivan, J.P., 2016. A Numerical model of Rotorua Geothermal Field. Geothermics, 60, 105-125.

Ripperda, N., Bodvarsson, G.S., Lipmann, M.J., Cuellar, G., Escobar, C., 1991. An exploitation model and performance predictions of the Ahuachapan geothermal field, El Salvador. Geothermics, 20, 4, 181-196.

Romagnoli, P., Arias, A., Barelli, A., Cei, M., Casini, M., 2010. An updated numerical miodeling of the Larderello-Travale geothermal system, Italy. Geothermics, 39, 292-313.

Rutqvist, J.,Wu, Y.-S., Tsang, J-F., Bodvarsson,G., 2002. A modeling approach for analsis of coupled multiphase flow, heat transfer and deformation in fractured porous rock. International Journal of Rock Mechanics and Mining Sciences 39, 429 442.

Sakagawa, Y., Aoyama, K., Ikuechi, K., Takahashi, M., Kato, O., Doi, N., Tosha, T., Ominato, T., Koide, K., 2000. Natural state simulation of the Kakkonda geothermal field, Japan., Proceedings of World Geothermal Congress, Kyushu-Tohuku, Japan, May 28-June 10, 2000, 2839-2844.
Sakagawa, Y., Takahashi, M., Hanano, M., Ishido, T., Demboya, N., 1994. Numerical simulation of the Mori geothermal field, Japan. Proceedings of the $19^{\text {th }}$ Workshop on Geothermal Reservoir Engineering, Stanford University, Stanford, California, 18-20 January 1994.

Sanchez, U.P., 1997. Los Humeros geothermal field, Mexico, Trans geothermal Resources Counc. 21, 435-439.

Sanyal, S.K., Antunez, E.U., Abe, M., Nakanishi, S., 1990. Numerical modeling of a mature high temperature geothermal reservoir; a case history from the Onikobe field, Miyagi prefecture, Japan. Transactions Geothermal Resources Council 14, 1339-1345.

Sanyal, S.K., Pham, M., Iwata, S., Suzuki, M., Inoue, T., Yamada, K., Futagoishi, M., 2000. Numerical simulation of the Wasabizawa geothermal field, Akita Prefecture, Japan, Proceedings of World Geothermal Congress, Kyushu-Tohuku, Japan, May 28-June 102000 2189-2194.

Seol, Y., Lee, K.K., 2007. Application of TOUGHREACT to performance evaluations of geothermal heat pump systems. Geosciences Journal, 11, 1, 83-91.

Sippel, J., Fuchs, S., Cacace, M., Braatz, A., Kastner, O., Huenges, E., Scheck-Wenderoth, M., 2013. Deep 3D thermal modelling for the city of Berlin (Germany). Environmental Earth Sciences, 70, $8,3545-3566$.

Soboleva, E., 2017. Numerical Simulation of Haline Convection in Geothermal Reservoirs, International Conference Problems of Thermal Physics and Power Engineering (Ptppe-2017), Journal of Physics Conference Series, 891, Natl Res Univ, Moscow Power Engn Inst, Moscow, RUSSIA, OCT 09-11, 2017, UNSP 012105.

Sonney, R., Vuataz, F.D., 2009. Numerical modelling of Alpine deep flow systems: a management and prediction tool for an exploited geothermal reservoir (Lavey-les-Bains, Switzerland). Hydrogeology Journal, 17, 3, 601-616.

Sta. Ana, F.X.M., Saw, V.S., Molina, P.O., Aleman, E.T., Canete, G.F., Hingoyon, C.S., Sarmiento, Z.F., 1999. Increased production load at 
Tngonan geothermal field, Philippines: Reservoi response and field management strategies. Proc of the 24 th workshop on geothermal reservoir engineering, Stanford, California (USA), 25-27 January 1999, 11-16.

Strobel, C.J., 1993. Buffalo field, Philippines: Reservoir modeling for prediction of limits to sustainable generation. Proc of the 18 th workshop on geothermal reservoir engineering, Stanford, California (USA), 26-28 January 1993, 5-10.

Suarez, Arriaga, M.C., Samaniego, V. F., Rodrigez, F., 1996. Some mismathces occurred when simulating fractured reservoir as homogeneous porous media. Proceedings of the $21^{\text {st }}$ Workshop on Geothermal Reservoir Engineering, Satnford University, Stanford, California, 26-28 January 1996, 179-186.

Suryadarma, D., T., Zuhro, A.A., Yani, A., 2010. Sustainable development of the Kamojang geothermal field. Geothermics, 39, 391-409.

Swenson, D., Schroeder, R., Shinohara, N., Okabe, T., 1999. Analyses of the Hijiori long term circulation test. Proceedings of the $24^{\text {th }}$ Workshop on Geothermal Reservoir Engineering, Stanford University, Stanford, California, 25-27 January 1999, 344-351.

Todesco, M., 1995. Modeling of the geothermal acticity at Vulcano (Aeolian Islands, Italy). Proceedings World Geothermal Congress'95, Florence, 18-31 May 1995, 1309-1315.

Tokita, H., Yahara, T., Kitakoga, I., 1995. Cooling effect and fluid behavior due to reinjected hot water in the Hatchobaru geothermal field, Japan. Proceedings World Geothermal Congress '95, Florence, 18-31 May 1995, 1869-1874.

Thomas, L.K., Pierson, R.G., 1978. 3-Dimensional Geothermal Reservoir Simulation. Society of Petroleum Engineers Journal, 18, 2, 151-161.

Toronyi, R.M., Farouq Ali, S.M., 1977. Two-phase, two-dimensional simulation of a geothermal reservoir. Soc. Pet. Eng. J., 17, 171-183.
Turali, E.Y., Simsek, S., 2017. Conceptual and 3D simulation modeling of the Sorgun hydrothermal reservoir (Yozgat,Turkey). Geothermics, 66, 85100 .

Vedova, D.B., Vecellio, C., Bellani, S., Tinivella, U, 2008. Thermal modeling of the Larderello geothermal field (Tuscany Italy). International Journal Earth Science (Geol Rundsch); 97, 317332.

Weijermars, R., Zuo, L., Warren, I., 2017. Modeling reservoir circulation and economic performance of the Neal Hot Springs geothermal power plant (Oregon, USA): An integrated case study, Geothermics, 70, 155-172.

White, S.P., Kissling, W.M., McGuinnes, M.J., 1997. Models of the Kawerau geothermal reservoir. Transactions Geothermal Resources Council, $21,33-40$.

Wijaya, I, Purqon, A., 2017. Simulation of Fluid Flow and Heat Transfer in Porous Medium Using Lattice Boltzmann Method, International Conference on Energy Sciences (Ices 2016), Bandung, Indonesia, Jul 25-27, 2016, Book Series: Journal of Physics Conference Series, Ed., Waris, A; Shin, B; Kondo, M; Buys, YF; Irwanto, D; Pramuditya, S., 877, Article Number: UNSP 012056.

Williamson, K. H., 1990. Reservoir simulation of The Geysers geothermal field. Proceedings of the 15 th Workshop on Geothermal Reservoir Engineering, Stanford University, Stanford, California, 23-25 January 1990, 113-123.

Xing, H.L., Liu, Y., Gao, J.F., Chen, S.J., 2015. Recent development in numerical simulation of enhanced geothermal reservoirs. Journal of Earth Science, 26, 1, 28-36.

Xu, T.F., Feng, G.H., Hou, Z.Y., Tian, H.L., Shi, Y., Lei, H.W., 2015. Wellbore-reservoir coupled simulation to study thermal and fluid processes in a $\mathrm{CO}_{2}$-based geothermal system: identifying favorable and unfavorable conditions in comparison with water. Environmental Earth Sciences, 73, 11, 6797-6813. 
Karahanoğlu

Xu; T., Pruess, K., 2001. Modeling multiphase nonisothermal fluid flow and reactive geochemical transport in variably saturate fractured rocks: 1 . Methodology. American J. Sci. 301(1), 16-33.

Yahara, T., Tokita, H., 2010. Sustainability of the Hatchobaru geothermal field, Japan. Geothermics, 39, 382-390.

Yano, Y., Ishido, T., 1995. Numerical modeling of the evolution of two phase zones under a fractured cap rock. Geothermics, 24, 4, 507-521.

Yasukawa, K., Ishido, T., 1990. Numerical modeling of the Onikobe caldera hydrothermal system, northeastern Honshu, Japan. Transactions Geothermal Resources Council 14, 1347-1355.

Yeh, H.D., Yang, S.Y., Li, K.Y., 2012. Heat extraction from aquifer geothermal systems, International Journal for Numerical and Analytical Methods in Geomechanics, 36, 1, 85-99.

Yoğurtcuoğlu, A., 2016. Integrated 3-D finite element simulation of the Edremit Geothermal Field. Yüksek Lisans tezi Orta Doğu Teknik Üniversitesi, Ankara.

Zaher, M.A., Saibi, H., El Noubi, M., Ghamry, E., Ehara, S., 2011. A preliminary regional assessment of the Gulf of Suez, Egypt. Journal of African Earth Sciences, 60, 117-132.

Zaher, M.A., Saibi, H., Nishijima, J., Fujimitsu, Y., Mesbah, H., Ehara, S., 2012. Exploration and assessment of the geothermal resources in the Hammam Faraun hot spring, Sinai Peninsula, Egypt. Journal of Asian Earth Sciences, 45, 256267.

Zang, Y.J., Li, Z.W., Yu, Z.W., Guo, L.L., Lin, X.P., $\mathrm{Xu}$, T.F., 2015. Evaluation of developing an enhanced geothermal heating system in northeast China: Field hydraulic stimulation and heat production forecast. Energy and Buildings, 88, $1-14$.
Zarrouk, S., O'Sullivan, M., Croucher, A., Mannington, W., 2007. Numerical modeling of production from the Poihipi dry steam zone; Wairakei geothermal system, New Zealand. Geothermics, 36, 289-303.

Zeng, Y., Tang, L., Wu, N.Y., Cao, Y.F., 2017. Analysis of influencing factors of production performance of enhanced geothermal system: A case study of Yangbajing geothermal field. Energy, 127, 218235.

Zeng, Y.C., Su, Z., Wu, N.Y., 2013. Numerical simulation of heat production potential from hot dry rock by water circulating through two horizontal wells at Desert Peak geothermal field. Energy, 56, 92-107.

Zhang, K., Wu, Y-S., Pruess, K., 2008. User's Guide for TOUGH2-MP- A massively parallel version of the TOUGH2 code. Report LBNL-315E, Lawrence Berkeley Laboratory, Berkeley CA, $108 \mathrm{pp}$.

Zhang, K., Lee, B.H., Ling, L.L., Guo, T.R., Liu, C.H., Ouyang, S., 2016. Modeling studies for production potential of Chingshui geothermal reservoir. Renewable Energy, 94, 568-578.

Zhao, Y.S., Feng, Z.J., Feng, Z.C., Yang, D., Liang, W.G., 2015. THM (Thermo-hydro-mechanical) coupled mathematical model of fractured media and numerical simulation of a 3D enhanced geothermal system at $573 \mathrm{~K}$ and buried depth 6000-7000 M. Energy, 82, 193-205.

Zyvoloski, G. 1983. Finite element methods for geothermal reservoir simulation. International Journal for Numerical and Analytical Methods in Geomechanics, 7: 75-86. 